NBSIR 79-1719

\title{
Statistics of Household Microwave Oven Use
}

Alan D. Davies

John V. Fechter

Consumer Sciences Division

Center for Consumer Product Technology

National Engineering Laboratory

National Bureau of Standards

Washington, D.C. 20234

January 1979

Final Report

Prepared for

National Bureau of Standards

and

Office of Conservation

Department of Energy

Washington, D.C. 20001 



\section{STATISTICS OF HOUSEHOLD MICROWAVE OVEN USE}

Alan D. Davies

John V. Fechter

Consumer Sciences Division

Center for Consumer Product Technology

National Engineering Laboratory

National Bureau of Standards

Washington, D.C. 20234

January 1979

Final Report

Prepared for

National Bureau of Standards

and

Office of Conservation

Department of Energy

Washington, D.C. 20001

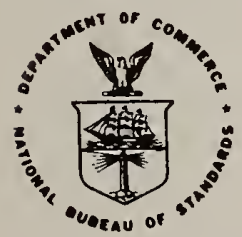

U.S. DEPARTMENT OF COMMERCE, Juanita M. Kreps, Secretary Jordan J. Baruch, Assistant Secretary for Science and Technology

NATIONAL BUREAU OF STANDARDS, Ernest Ambler, Director 



\section{ACKNOWLEDGMENTS}

The authors wish to thank Mr. James Cole for his assistance in data reduction and Mrs. Ruth Warnock

for typing the completed manuscript. 

Acknowledgments . . . . . . . . . . . . ii

Abstract . . . . . . . . . . . . 1

Introduction . . . . . . . . . . . . . . . 1

Principal Definitions . . . . . . . . . . 3

Results . . . . . . . . . . . . . 4

Servings and Eatings . . . . . . . . . 6

MWO-Owner Eatings and Servings ....... . 8

Food Selection............ 8

Task Displacement by MWO's......... . 13

Demographic Analyses . . . . . . . . . 17

Income Level . . . . . . . . . 17

Household Size . . . . . . . . 17

Season . . . . . . . . . . . 19

Discussion . . . . . . . . . . . . . 19

References ................ . 21

Appendix A. Demographic Characteristics and Comparisons

of the Sampled Households . . . . . . 22

Appendix B. Contingency Tables . . . . . . . 30

Appendix C. Household and Meal Characteristic Profiles

for MWO-owners and Non-Owners . . . . 32

Appendix D. Permission to Release Restricted Data . . 51 
Table

No.

Page

1 Total Servings (S), total Eatings (E), and

Eatings per Serving $(\mathrm{E} / \mathrm{S})$. . . . . . . . . 5

2 Comparisons of Servings and Eatings as a

function of MWO ownership and food

categorization . . . . . . . . . . . . 7

3 Servings and Eatings for Report B foods;

comparison of: Leftover and Total foods for

MWO households . . . . . . . . . . . 8

4 Comparison of MWO-Owner and non-owner households for preparing five different categories

of food

5 Comparison of MWO-owners and non-owners for

the pre-preparation status of foods cooked

or heated . . . . . . . . . . . . . 11

6 A list of major food groupings where the difference in Eatings or Servings between MWO-owners and non-owners was 5 percent or more . . . . . . . . . . . . . . . .

7 Comparison of selection rates of MWO's and other devices for major food groups . . . . . . . 14

8 Device preferences by a sample of seven non-owner cooks for preparing the AHAM menu . . 16

9 Projected displacement of cooking tasks by MWO's (event counts rounded to nearest ten) . . . .

10 Total Servings and Eatings per household ( $\mathrm{HH})$, all foods, by $\mathrm{HH}$ income, $\mathrm{HH}$ size and season. .

Al Selected characteristics for the U.S. population and the sample of 2000 households used for the Fifth MRCA Menu Census . . . . . . . . . . 23

A2 Comparison of MWO and non-MWO households on the basis of demographic characteristics . • . 26

Cl Owner household and meal characteristics for foods cooked or heated in MWO's . . . . . . 35 
Table

No.

Page

C2 Owner household and meal characteristics

for cooked or heated, but not in MWO's... . 39

C3 MWO-owner household and meal characteristics for all foods cooked or heated . . . . . 43

C4 Non-owner household and meal characteristics for all foods cooked or heated . . . . . 47 

Alan D. Davies

John V. Fechter

\section{ABSTRACT}

The purpose of the work reported here was to obtain statistical information on the use of Microwave Ovens (MWO's) in the home with respect to foods cooked or heated and the amount of MWO usage relative to other cooking devices. The work was funded jointly by the National Bureau of Standards and the Department of Energy. Data were provided by the Market Research Corporation of America (MRCA) from a national menu census survey conducted in 1975. NBS also conducted a very small survey on MWO displacement of other cooking devices.

From its 2000 household panel, MRCA identified 96 households that owned MWO's and selected a demographically matched set of 96 non-owner households. Aggregated data were provided on Servings (dishes prepared) and Eatings (persons partaking of a Serving) over a two week period for each household. The main findings from analyses of these data are:

- There was little difference between the owner and non-owner households in terms of total Eatings or Servings or in the proportions of leftovers eaten.

- In owner households, MWO's accounted for approximately 10 percent of the Servings and 9 percent of the Eatings.

- Leftovers accounted for 9 percent of the Eatings for both groups, and for 11 percent of owner Servings and 9 percent of non-owner Servings.

- MWO's were used by owners for 26 percent of leftover Servings and 23 percent of leftover Eatings.

\section{INTRODUCTION}

Congress initiated an energy efficiency improvement program for major household consumer products as part of its overall energy conservation program with its Energy Policy 
and Conservation Act, EPCA (1), followed by the Energy Conservation and Product Act (2). Kitchen ranges and ovens, including microwave ovens (MWO's) are among the products covered by the EPCA. Products covered by this program will bear point-of-sale labels informing consumers of energy efficiency and estimated annual operating cost.

Standardized tests and methods for computing efficiencies and operating costs have been prepared for all products covered by the EPCA. These test procedures include estimates of the average annual amount of consumer service performed by each product, which form the bases for the annual cost estimates. Since MWO's account for a large part of the market in cooking equipment and were relatively new at the start of the project, the Department of Energy (DOE) and the National Bureau of Standards (NBS) decided that a national survey was needed to answer certain questions about MWO usage. These questions were:

1. Do MWO owners differ from non-owners in the amounts or types of foods cooked or heated?

2. What share of the cooking and heating was actually done in MWO's during the test year by MWO owners, notwithstanding what could have been done?

3. A question added after the data assembly was committed was: How much of the cooking loads did the MWO's take over from the conventional cooking tops and from the ovens?

This report contains 1975 field survey information on the use of MWO's and other cooking equipment for the cooking and heating of foods. The data were collected by the Market Research Corporation of America (MRCA) as part of its periodic menu census program. MRCA has a nationwide panel of 2000 participating households that was statistically selected to be representative of the general U.S. population. (See Appendix A for details.) Their clients include many major corporations needing reliable statistical information on consumer use of goods and services. MRCA identified 96 owners of MWO's and a demographically matched sample of 96 non-owners as the subjects from its overall panel and abstracted the required information for the 192 households from its general data base. The analysis was done by NBS. In addition to providing information applicable to the energy conservation program, the results can be used as base year data for similar studies in the 
future and as background for laboratory studies of consumer behavior. Some additional data are provided beyond the immediate needs for the potential benefit of future investigators.

The raw data consist of detailed records from each household on foods cooked or heated during a period of two weeks, with these periods being distributed over a year to cover possible seasonal effects. MRCA excluded all cases not involving cooking or heating at home and provided two types of summary report. For Report $A$, records were examined to identify all food items prepared at least once in a MWO. Then for all such food items, MRCA reported how often the food was prepared in a MWO, how often it was cooked or heated by MWO households but not in the MWO, and how often the same item was prepared by the matched, non-Mwo households. A parallel report (Report B) was also prepared covering all items prepared by the 192 households, whether or not an MWO had been used.

Data aggregations were selected by NBS to avoid the presentation of unneeded detail, to protect proprietary rights reserved by the contractor, and to minimize artificialities that might have arisen due to MRCA's particular data structure or detailed breakdown of food item classification. Limitations on the release of MRCA data are given in Appendix D.

Many questions about MWO cooking can be addressed using these data. However', this report is limited to matters bearing on energy-related test procedures. It was not the objective of the study to test predetermined hypotheses about cooking practices. Rather, the objective was to explore these practices as they existed in 1975, and to relate them to the energy efficiency improvement program and to current needs of the Department of Energy (DOE).

\section{PRINCIPAL DEFINITIONS}

Servings and Eatings are specialized terms used by the contractor throughout their reports and tabulations, and have been left intact by the authors.

Servings, S: A serving means an occasion in which a cooking device (MWO or other, usually a conventional range) 
is used to prepare a given dish, whether it is to be consumed by one or more eaters.

Eatings, E: There is one "eating" for each person that partakes of a given dish. Thus, a single serving of roast beef might generate five "eatings" and a serving of coffee more than one eating.

Microwave Candidate Foods Report, A: A food category qualified for inclusion in the "A" report if and only if it was prepared at least once in an MWO by a member of the 96 household owner panel. If a category qualified, the corresponding non-owner data were also provided. Items eaten without being cooked or heated at home are not included in Report A or B.

All Foods Report, B: This report covers all foods cooked or heated at home, whether an MWO was ever used for the item or not.

First-Time Preparation: This refers to the initial preparation of a dish, with the alternative being preparation as a left-over, L/O.

Left-Over: This refers to a cooked or heated leftover.

\section{RESULTS}

The principal findings are derived from analysis of the grand totals of Eatings and Servings presented in Table 1. Many of the conclusions follow directly from examination of this and other tables. Readers are cautioned against making unduly refined conclusions from these data, since changes from a very few households could significantly affect some results.

There is no general rule for deciding the importance of any given ratio, overall factor or comparative difference; readers must decide this for each application. Because neither consumer safety nor great economic risk is involved; it is suggested that strong evidence (e.g., in the first or second significant figures) be required before readers can make important distinctions. The main comparisons are between MWO owners and non-owners to identify similarities and differences in cooking behavior that are associated with MWO ownership, and between the A report (MWO-Candidate 


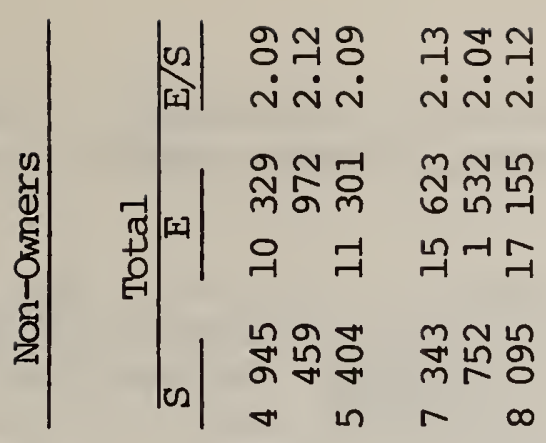

वิ

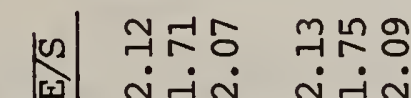

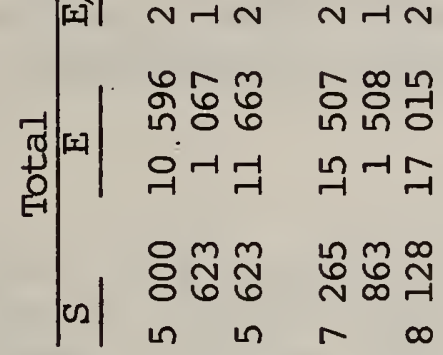

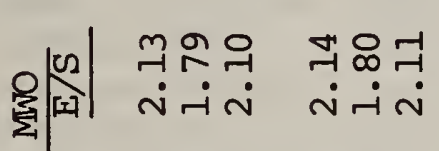

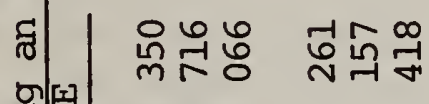

격 व

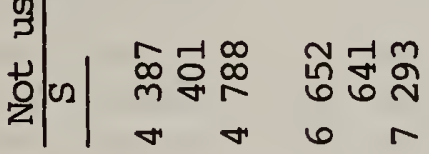

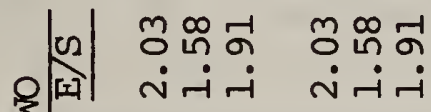

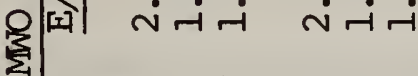

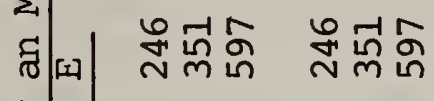

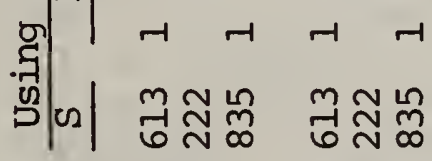

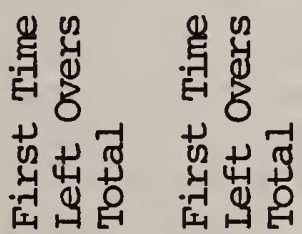

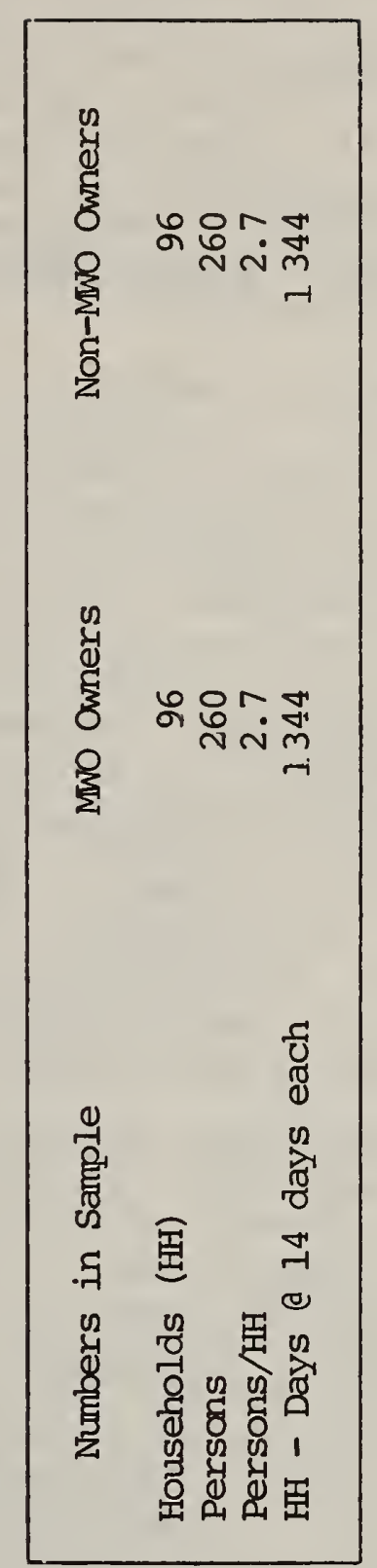

-

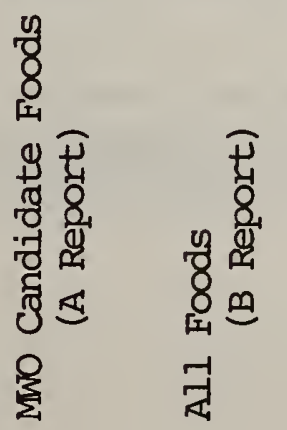


Foods) and the B report (All Foods) bearing on matters of food selection.

The numbers of Eatings generally indicate the amount of food cooked, and the number of servings generally indicate the number of cooking events. Servings overestimate the number of cooking events to the extent that more than one dish was cooked at the same time in an oven. A low ratio of Eatings per Serving indicates more cooking and eating alone (for example, homemaker lunches). The following discussion involves a closer examination of relationships seen in Table 1 and elsewhere.

Servings and Eatings:

As seen in Table 2, total Eatings and total servings for "all foods" were almost identical for owners and nonowners, in spite of the fact that skipping meals, eating away from home, snacking habits, and the number of hot dishes per meal could vary widely among households. Chisquared $\left(x^{2}\right)$ statistics are provided for more formal studies of statistical independence between attribute systems. The percentage, $P$, associated with each reported $x^{2}$ indicates the probability that a $x^{2}$ at least that large could have occurred by chance, given no real relationship between attribute systems. (An explanation of contingency tables and $x^{2}$ calculations is provided in Appendix $B$, along with an example in which the relative tendencies of owners and nonowners to use leftovers are examined. No significant difference was found.)

Regarding the MWO-Candidate Foods (Report A), it should be noted that a food became a member of this group only if it was cooked or heated at least once in a MWO. Given this selection process, it is not surprising to find a slight bias for MWO-owners to prepare and eat more foods from the $A$ group than non-owners. For owners, 31 percent of both Servings and Eatings came from food types never cooked in MWO's, while 33 percent of the servings and 34 percent of the Eatings by non-owners came from these same groups. Generally, owners and non-owners display quite similar frequencies of preparing MWO-Candidate Foods and All Foods with respect to the number of First Time, Leftover, and Total Eatings and Servings.

Referring directly to the All Foods (Report B) group in Table 1, Leftovers accounted for 9 percent of the Eatings for both owners and non-owners and for 9 percent of the 
Table 2. Comparisons of Servings and Eatings as a function of MwO ownership and food categorization.

\begin{tabular}{|c|c|c|c|c|}
\hline & & igs & & hgs \\
\hline & Fir & Time & & Time \\
\hline & Mwo-Owners & Non-Owners & MWO-Owners & Non-Owners \\
\hline $\begin{array}{l}\text { MWO Candidate } \\
\text { Foods }\end{array}$ & 5000 & 4945 & 10596 & 10329 \\
\hline All Foods & $\begin{array}{c}7265 \\
x^{2}=0.70\end{array}$ & $\begin{array}{cc}7 & 343 \\
75 \% & d f=1\end{array}$ & $\begin{array}{c}15507 \\
x^{2}=3.40,\end{array}$ & $\begin{array}{r}15623 \\
\simeq 4 \%, d f=1\end{array}$ \\
\hline
\end{tabular}

Left Overs

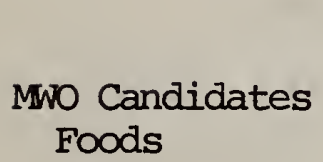

All Foods

\begin{tabular}{|cc|}
\hline MwO-Owners & Non-Owners \\
623 & 459 \\
863 & 752 \\
$\chi^{2}=4.49, \mathrm{p} \simeq 3 \%$, df $=1$ \\
\hline
\end{tabular}

Total

\begin{tabular}{|cc|}
\hline Mwo-Owners & Non-Owners \\
5623 & 5404 \\
8128 & 8095 \\
$\chi^{2}=2.09, \mathrm{p} \simeq 16 \%, \mathrm{df}=1$ \\
\hline
\end{tabular}

\section{Left Overs}

$\begin{array}{cr}\text { Mwo-Owners } & \text { Non-Owners } \\ 1067 & 972 \\ 1508 & 1532 \\ \chi^{2}=3.62, p \simeq 6 \%, \text { df }=1\end{array}$

\section{Mwo Candidates Foods}

All Foods 
Servings for non-owners versus 11 percent of the Servings for owners. In other words, MWO-owners utilize about the same number of Leftovers as do non-owners. The $\chi^{2}$

computation example in Appendix B indicates a very close similarity between Eatings by owners and non-owners with respect to the proportion of first time and leftover foods.

MWO-Owner Eatings and Servings:

MWO's accounted for 10 percent of the total Servings and 9 percent of the total Eatings for the owner group, indicating a tendency to use MWO's for smaller quantities of food at a time.

Leftovers prepared in MWO's accounted for 26 percent of all Leftover Servings and 23 percent of all Leftover Eatings in MWO households (see Table 3). Preparing Leftovers accounted for 27 percent of all MWO uses in terms of Servings and 22 percent in terms of Eatings.

Table 3. Servings and Eatings for Report B foods; comparison of Leftover and Total foods for MWO households.

Servings

Prepared in MWO

Leftovers

Total
All Heated or Cooked Foods

222

835
863

$8 \quad 128$

Eatings

\begin{tabular}{l} 
Prepared \\
in MWO \\
\hline
\end{tabular}

351

1597
All Heated or Cooked Foods

1508

$\begin{array}{ll}17 & 015\end{array}$

Food Selection:

NBS and others have observed differences in dielectric constants among food types which affects energy absorption rates. Also, roles are suggested by some for the use of standardized menus in the development of some numerical factors used in the test procedures. Data on food selection are presented in the event that DOE or other interested parties determine that food type has an important bearing on MWO cooking and heating efficiency for test procedure purposes. MRCA reported data involving well over a hundred primary and subclassifications of food. Many of these classifications reflect the special requests of commercial clients and are much too detailed for present purposes. At the request of NBS, MRCA regrouped the data using two separate methods. Cross tabulations were then generated between these groupings and selected demographic 
characteristics of the subject groups. The grouping methods were:

METHOD I

\author{
ritle \\ Description \\ Liquids \\ Beverages, soup, baby food, gravy \\ and sauces \\ Animal Protein Beef, lamb, fish, poultry, processed \\ meats \\ Fruits \& Vegs. Vegetables (including potatoes), fruits \\ Hot Dishes \\ Dishes with eggs, pasta, rice, cereal, \\ cheese \\ Baked Items \\ Breads, cakes, cookies, pies \\ Other (I) \\ Remaining items not covered above
}

METHOD II

Title

Description

Raw

Ingredients not previously cooked nor frozen at preparation time

Convenience

Substantially pre-cooked (generally needs only heating or browning)

Frozen

Initially frozen at preparation time

Other (II)

Remaining items not covered above

Tendencies of owners and non-owners in the Serving and Eating of various food groups are shown in Tables 4 and 5 . Omitting consideration of the "Other" categories, difference percentages were computed by dividing the larger number of Eatings or Servings by the smaller number for each food group. Those cases where the difference was 5 percent or more are shown in Table 6 . Owners of MWO's showed a consistent tendency to cook or heat baked goods more than non-owners. 
Table 4. Comparison of MwO-owner and non-owner households for preparing five different categories of food.

AII, FOODS (Report B)

Servings

Food Group

Liquids

Animal Protein

Fruits \& Vegs.

Hot Dishes

Baked Items

Subtotal

Other

Total

MwO-Owners Non-Owners

2778

1249

2844

1160

1208

1249

1207

$\frac{1501}{7937}$

1314

1314

$\frac{191}{8128}$

$\frac{208}{8095}$

Eatings

MWO-Owners Non-Owners

Considering only

the five main

food groups

$$
x^{2}=16.31, p<1 \% \text { df }=4 \quad x^{2}=27.95, p<1 \%
$$

Considering all

five main food groups and all "other" foods

$$
x^{2}=17.04, p<1 \% \text { df }=5 x^{2}=45.48, p<1 \%
$$

MICROWAVE CANDIDATE FOODS (Report A)

\section{Servings}

Food Group

Liquids

Animal Protein

Fruits \& Vegs.

Hot Dishes

Baked Items

Subtotal

Other

Total

Considering only

the five main

food groups

Considering all

five main food

groups and all

"other" foods
MwO-Owners Non-Owners

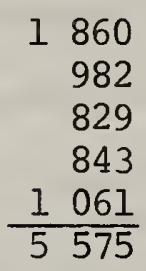

$\frac{48}{5623}$

\section{Eatings}

\begin{tabular}{|c|c|}
\hline 3. 057 & 3282 \\
\hline 2414 & 2241 \\
\hline 2222 & 2297 \\
\hline 1876 & 1698 \\
\hline 2004 & 1677 \\
\hline 11573 & $\overline{11195}$ \\
\hline & 106 \\
\hline 1166 & \\
\hline
\end{tabular}

Mwo-Owners Non-Owners

$$
x^{2}=28.44, p<1 \% \text { df }=4 \quad x^{2}=47.31, p<1 \%
$$

$$
\chi^{2}=28.44, p<1 \% \text { df }=5 \quad \chi^{2}=49.19, p<1 \%
$$


Table 5. Comparison of MwO-owners and non-owners for the pre-preparation status of foods cooked or heated.

\begin{tabular}{|c|c|c|c|c|c|}
\hline \multirow{3}{*}{$\begin{array}{l}\text { Pre-Preparation } \\
\text { Status }\end{array}$} & \multicolumn{3}{|c|}{ ALL FOODS (Report B) } & \multirow{2}{*}{\multicolumn{2}{|c|}{ Eatings }} \\
\hline & \multicolumn{2}{|c|}{ Servings } & & & \\
\hline & MWO-Owners & Non-Owners & & MWO-Owners & Non-Owners \\
\hline Raw & 3015 & 3026 & & 7515 & 7570 \\
\hline Convenience & 2669 & 2548 & & 5289 & 5120 \\
\hline Frozen & 577 & 663 & & 1431 & 1649 \\
\hline Subtotal & $\overline{6261}$ & $\overline{6237}$ & & 14235 & $\overline{14 \quad 284}$ \\
\hline $\begin{array}{c}\text { Other (II) } \\
\text { Total }\end{array}$ & $\begin{array}{ll}1 & 867 \\
8 & 128\end{array}$ & $\begin{array}{ll}1 & 858 \\
8 & 095\end{array}$ & & $\frac{2780}{17015}$ & $\frac{2871}{17155}$ \\
\hline $\begin{array}{l}\text { Considering only } \\
\text { the three main } \\
\text { pre-preparation } \\
\text { status groups }\end{array}$ & $\chi^{2}=8.75$, & $p<1 \%$ & $d f=2$ & $\chi^{2}=18$ & $00, \mathrm{p}<1 \%$ \\
\hline $\begin{array}{l}\text { Considering the } \\
\text { three main pre- } \\
\text { preparation status } \\
\text { groups and the } \\
\text { "other" category }\end{array}$ & $x^{2}=8.67$, & $\simeq 4 \%$ & $d f=3$ & $x^{2}=18$ & \\
\hline
\end{tabular}

MICROWAVE CANDIDATE FOODS (Report A)

Pre-Preparation Status

\section{Servings}

MWO-Owners Non-Owners

2213

1796

$\frac{301}{4310}$

$\frac{1313}{5623}$ \begin{tabular}{l}
2085 \\
$1 \quad 609$ \\
$\quad 259$ \\
\hline 3953
\end{tabular}

$\frac{1451}{5404}$

\section{Eatings}

Mwo-Owners Non-Owners

\begin{tabular}{rrr}
5426 & 5180 \\
3653 & 3329 \\
746 & $\quad 686$ \\
\hline 9825 & 9195
\end{tabular}

$\frac{1838}{11663}$

Considering only

the three main pre-preparation status groups

$$
\chi^{2}=1.73, p \simeq 46 \% \quad \mathrm{df}=2 \quad \chi^{2}=2.39, \mathrm{p} \simeq 30 \%
$$

Considering the three main prepreparation status groups and the "other" category $\chi^{2}=19.78, p<1 \% \quad \mathrm{df}=3 \quad \chi^{2}=35.69, \mathrm{p}<1 \%$ 
Table 6. A list of major food groupings where the difference in Eatings or Servings between MWO-owners and non-owners was 5 percent or more.

\begin{tabular}{llll} 
& Percentage & Source of & $\begin{array}{c}\text { Type of Household with } \\
\text { the Iarger Number of }\end{array}$ \\
Food Group & Difference & Difference & Eatings or Servings \\
\hline
\end{tabular}

All Foods

(Report B)

Fruits \& Vegs.

$6 \%$

Hot Dishes

Baked Items

Baked Items

Frozen

Frozen

Microwave Candidate

Foods (Report A)

Liquids
Liquids

Animal Protein

Animal Protein

Hot Dishes

Hot Dishes

Baked Items

Baked Items

Raw

Convenience

Convenience

Frozen

Frozen
$5 \%$

$14 \%$

: $14 \%$

$15 \%$

$15 \%$

$7 \%$

$7 \%$

$10 \%$

$8 \%$

$10 \%$

$10 \%$

$23 \%$

$19 \%$

$6 \%$

$12 \%$

$10 \%$

$16 \%$

$9 \%$
Eatings

Servings

Servings

Eatings

Servings

Eatings

Servings

Eatings

Servings

Eatings

Servings

Eatings

Servings

Eatings

Servings

Servings

Eatings

Servings

Eatings non-owners

non-owners

owners

owners

non-owners

non-owners

non-owners

non-owners

owners

owners

owners

owners

owners

owners

owners

owners

owners

owners

owners 
In the case of frozen foods (which accounted for less than 10 percent of all Servings or Eatings) non-owners prepared frozen foods on the "all foods" list more often, whereas owners favored prepared frozen foods on the "microwave candidate" list. This reversal in relative activity for microwave candidate foods is due in part to the way the candidate list was developed. A food could join this list only if one or more owners prepared the food at least once in a MWO. This selection procedure automatically biases the candidate list towards foods preferred by owners.

Based on data from the All Foods Report (Report B), Table 7 relates owner usage of MWO's relative to other cooking devices for the two sets of food groups. (In both cases, the "other" foods data were omitted.) Cooking device selection preferences are indicated by the ratio of foods prepared using other devices to foods prepared in an MWO. These ratios are found by dividing the number of non-MWO events by the corresponding number of MWO events

for each food group. The ratios may be considered as the odds against preparation in an MWO, and when compared with the odds given on the Total lines the ratios indicate owners' preference for using an MWO to cook foods in each group. MWO's were used less frequently than the average for liquids and for convenience foods.

Task Displacement by MWO's:

Questions that arose well after the survey was committed concerned the degree to which MWO's in combined microwave/conventional ranges take over tasks from conventional cook tops and ovens, and whether there are new cooking tasks performed in households having a MWO.

Although these questions cannot be answered directly from the survey data, an attempt was made to develop approximate answers.

The test procedure (4) contains two factors, $K$ for conventional ovens and I for conventional cooking tops, which are defined as the estimated fractions of input energy consumption due to microwave oven usage. It should be noted that $K$ and $I$ refer to input energy, whereas the MRCA data refer to output services to the consumer. specific data on quantities of foods prepared and eaten are not part of the MRCA data, nor are the cooking efficiencies of the devices involved. Hence, the ability to relate energy outputs to inputs is not as good as could be asked. 
Table 7. Comparison of selection rates of MiO's and other devices for major food groups.

\begin{tabular}{|c|c|c|c|c|c|c|c|c|c|c|}
\hline & & & & $\mathrm{FOOD} \mathrm{GI}$ & UPING MI & THOD I & & & & \\
\hline & & & Servi & ngs & & & & Eatin & & \\
\hline Food Group & M & $X M$ & $\begin{array}{l}\mathrm{XM} / \mathrm{M} \\
\text { Ratio } \\
\end{array}$ & MXM & $\begin{array}{l}\text { Percent } \\
\text { of MXM } \\
\text { Total } \\
\end{array}$ & M & $X M$ & $\begin{array}{l}\text { XIN/M } \\
\text { Ratio }\end{array}$ & MXM & $\begin{array}{l}\text { of } M \\
\text { Tot }\end{array}$ \\
\hline Liquids & 158 & 2620 & 17 & 2778 & 35.0 & 210 & 4297 & 20 & 4507 & \\
\hline Animal Protein & 202 & 1047 & 5 & 1249 & 15.7 & 418 & 2632 & 6 & 3050 & \\
\hline Fruits \& Vegs. & 173 & 987 & 6 & 1160 & 14.6 & 406 & 2686 & 7 & 3092 & \\
\hline Hot Dishes & 156 & 1093 & 7 & 1249 & 15.7 & 298 & 2489 & 8 & 2787 & \\
\hline Baked Items & 127 & 1374 & i1 & 1501 & 18.9 & 237 & 2866 & 12 & 3103 & \\
\hline Total* & 816 & 7121 & 11.5 & 7937 & 100.0 & 1569 & 14970 & 9.5 & 16539 & 100 \\
\hline $\begin{array}{l}\text { Considering } \\
\text { the food } \\
\text { group }\end{array}$ & $x^{2}$ & I) & 49.5 & & df & $x^{2}(1-x)$ & $\mathrm{XM}$ ) & .4 & 1 & $=$ \\
\hline
\end{tabular}

\section{FOOD GROUPING METHOD II}

\begin{tabular}{|c|c|c|c|c|c|c|c|c|c|c|}
\hline & & & & & & & & & & \\
\hline & & & Servi & ings & & & & Eatin & & \\
\hline Food Group & M & $\mathrm{XM}$ & $\begin{array}{l}\text { XM/M } \\
\text { Ratio }\end{array}$ & MXM & $\begin{array}{c}\text { Percent } \\
\text { of MXMM } \\
\text { Total }\end{array}$ & M & $\mathrm{XM}$ & $\begin{array}{l}\text { XM/M } \\
\text { Ratio }\end{array}$ & MXMM & $\begin{array}{l}\text { Percen } \\
\text { of MXM } \\
\text { Total }\end{array}$ \\
\hline Raw & 396 & 2619 & 7 & 3015 & 48.2 & 811 & 6704 & 8 & 7515 & 52.8 \\
\hline Convenience & 249 & 2420 & 10 & 2669 & 42.6 & 500 & 4789 & 10 & 5289 & 32.2 \\
\hline Frozen & 94 & 483 & 5 & 577 & 9.2 & 180 & 1251 & 7 & 1431 & 10.0 \\
\hline Total* & $\overline{739}$ & $\overline{5522}$ & 7.5 & 6261 & $\overline{100.0}$ & 1491 & $\overline{12744}$ & 9.5 & 14235 & 100.0 \\
\hline $\begin{array}{l}\text { Considering } \\
\text { the food }\end{array}$ & & & & & & & & & & \\
\hline group & $x^{2}(1$ & $\mathrm{XM})=$ & $=31.9$, & $p<1 \%$ & $d f=4$ & $\chi^{2}(M$ & $X M)=$ & $.0, \mathrm{~F}$ & $1 \%$ & $=4$ \\
\hline
\end{tabular}

$M=$ Prepared using an MwO.

$X M=$ Prepared not using an MWO.

$M X M=M+X M$

*"Other" categories were omitted. 
The test procedure (4) also specifies annual useful cooking energy outputs for use in computing estimated annual operating costs. The energy outputs are given as 1108000 Btu's per year for conventional ranges and 1054000 Btu's per year for microwave/conventional ranges. The difference is based on a six-family survey by a manufacturer. With no MWO present, energy consumption was metered for two years to obtain baseline data. Then, after allowing three months for the households to become familiar with new counter top MWO's, energy consumption was metered for another nine months. Annualized average energy consumption was lower when the MWO's were in place. These data were converted to values of cooking energy output and corresponding $K$ and $L$ values.

Because such meter data were unavailable for the MRCA data, the problem of taking over cooking tasks was approached by assuming that each competing cooking device would lose tasks and energy input requirements to the MWO in the same proportion, and that the numbers of Eatings and Servings would remain unchanged when the MWO was introduced. On this basis, the data in Table 3 would apply, indicating that 10 percent of the total servings and 9 percent of the total Eatings were taken over by the MWO from each of the other competing devices. Using Eatings as the suggested basis of displacement, both $K$ and $I$ in the test procedure (4) would be 0.91 instead of $K=0.82$ and $L=0.85$ in the current test procedure (4).

An alternate approach would be to identify the frequency that each non-MWO cooking device is normally used and to estimate MWO displacement by food group. In the absence of such data seven experienced cooks at NBS were asked to select cooking devices they would use to prepare meals from the Kitchen Range Test Menu developed in 1975 by the Association of Home Appliance Manufacturers (AHAM). (All seven happened to be non-owners.) For each dish on the AHAM menu that needs cooking or heating, each respondent identified from among cooking top, conventional oven and other non-MWO devices the cooking device normally used or that would be used. The data were compiled and the dishes allocated among the Liquids, Animal Protein, Fruits \& Vegetables, Hot Dishes, and Baked Items food groups. The results are shown in Table 8 . 
Table 8. Device preferences by a sample of seven non-owner cooks for preparing the AHAM menu.

\begin{tabular}{lcccc} 
& \multicolumn{2}{c}{ Fraction Assigned to } \\
Food Group & Cooking Top & Oven & Other Device \\
\cline { 2 - 2 } Liquids & 0.95 & & 0.00 & 0.05 \\
Animal Protein & 0.50 & 0.50 & 0.00 \\
Fruits \& Vegs. & 1.00 & 0.00 & 0.00 \\
Hot Dishes & 0.80 & 0.20 & 0.00 \\
Baked Items & 0.20 & 0.70 & 0.10
\end{tabular}

The preferences in Table 8 were then applied to the data for all foods cooked by non-owners (Table 4), and to the owner data on non-MWO cooking in Table 9 on the basis that owners who elected not to use an MWO on a particular occasion would have device-selection preferences similar to non-owners for non-MWO cooking. The resulting allocations are shown in Table 9.

Table 9. Projected displacement of cooking tasks by MWO's (event counts rounded to nearest ten).

\begin{tabular}{|c|c|c|c|c|c|c|c|c|}
\hline \multirow[b]{2}{*}{ Cooking Events } & \multicolumn{4}{|c|}{ Servings } & \multicolumn{4}{|c|}{ Eatings } \\
\hline & $\begin{array}{l}\text { Cooking } \\
\text { Top }\end{array}$ & Oven & $\begin{array}{l}\text { Other } \\
\text { Device }\end{array}$ & Total & $\begin{array}{l}\text { Cooking } \\
\text { Top }\end{array}$ & Oven & $\begin{array}{l}\text { Other } \\
\text { Device }\end{array}$ & Total \\
\hline Non-owners & 5700 & 1790 & 270 & 7760 & 12090 & 4050 & 510 & 16650 \\
\hline $\begin{array}{l}\text { Owners, not in } \\
\text { MWO }\end{array}$ & 5150 & 1700 & 270 & 7120 & 10650 & 3820 & 500 & 14970 \\
\hline $\begin{array}{l}\text { Difference } \\
\text { (Frequency) }\end{array}$ & 550 & 90 & 0 & 640 & 1440 & 230 & 10 & 1680 \\
\hline $\begin{array}{l}\text { Percent } \\
\text { Displacement* }\end{array}$ & 9.6 & 5.0 & 0.0 & 8.2 & 11.9 & 5.7 & 2.0 & 10.1 \\
\hline
\end{tabular}

*Percent displacement $=$ (difference/non-owner cooking events) $\times 100$.

Using rounded values of the percentages of Eatings displacement, $\mathrm{K}$ (for ovens) would be 0.94 and $\mathrm{L}$ (for cooking tops) would be 0.88 . It should be noted that the MWO's in this survey were independent devices, whereas $K$ and $L$ apply to structurally combined devices. 
The question of additional cooking tasks being stimulated by MWO ownership was addressed inconclusively. Referring to the All Foods portion of Table 1, MWO owners reported 33 more Servings than non-owners $(8128-8095=33$, a 0.4 percent difference) and 140 fewer Eatings (17 015$17155=-140$, a 0.8 percent difference) than non-owners. It is not recommended that any important conclusions be based on these differences.

Demographic Analyses:

Demographic data at the All Foods level are provided in Appendix C. The title page of Report A lists all demographic arrangements of the data reported by MRCA. Corresponding data for All Foods were provided by the contractor in their Report B.

The data for Household Income, Household Size and Seasonal Totals were selected for analysis as being of potential interest for present DOE impact analysis programs. The data given in Table 10 are derived from Appendix $C$ by dividing the event counts by the particular numbers of households involved, yielding average events per household over a 14-day period. Caution against undue refinement is advised in interpreting these data because unusual behavior by one or two households could distort results, especially for some of the less populous cells. The data were examined for either the presence or absence of a systematic relationship with respect to the independent factor, since an indication either way may be useful in planning later work.

\section{Income level:}

No systematic variation of Servings as a function of income group is apparent unless the generally lower figures for the top income group might indicate more eating away from home. Eatings rise with income, showing little variation from the $\$ 10000$ to $\$ 25000$ income levels, and then a decline above $\$ 25000$.

Household Size:

Servings, Eatings and Eatings per Serving are smallest for one-person households. Servings rise slowly with increasing household size. Eatings rise in almost direct proportion to household size. 
o 5 N ஸ

Frmo/o

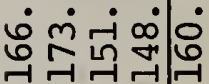

N N $\infty$ ம்

$66 \sim N m$

n $\infty \mathrm{n} / \mathrm{N}$

量

'ृ્డ

ซ్

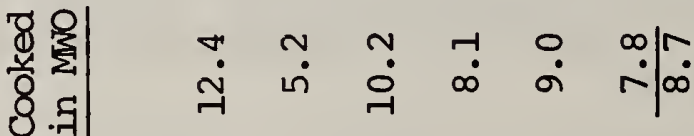

- I I $\stackrel{\text { กิ }}{\text { \% }}$ 어 $\stackrel{m}{r} \underset{r}{N}$ 우 $\infty$

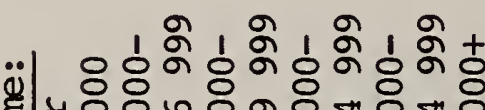
है।

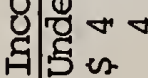

ㄴ.mm: ก่ $m m$ m.

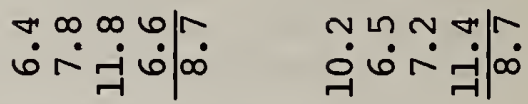

․ำ 늄ำ

ㄱํำ 기양

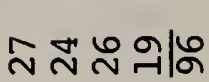

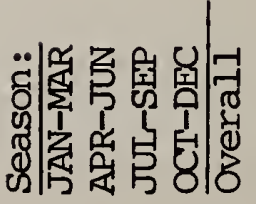

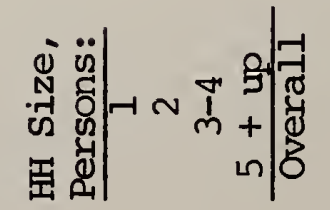

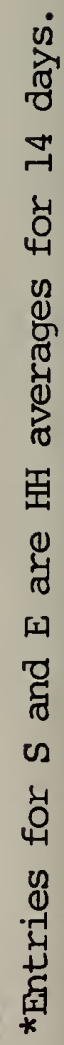


The cooking and the eating of cooked foods is least for both owners and non-owners during the summer months. Owners and non-owners did not display any consistent pattern for Eatings and Servings in the other seasons.

\section{DISCUSSION}

As described in Tables 1 and 2, MWO-owners behaved about the same as non-owners with respect to the total numbers of Eatings and Servings they cook or heat. Leftovers were eaten as often in both types of households. Considering overall frequencies of use, owners used MWO's proportionately more often than other devices when preparing leftovers.

Owners of MWO's rarely used their MWO's exclusively for preparing a given type of food. Instead, for most foods (approximately 90 percent of all Eatings and Servings), nonMWO cooking devices were selected. Several foods were prepared in MWO's fewer than ten times by the entire group of 96 MWO households, suggesting some amount of experimenting with MWO's.

Overall, only 10 percent of the cooked or heated food eaten in a MWO-household was prepared in an MWO. Roughly 10 percent of all household cooking tasks were displaced from conventional devices by MWO's.

One-third of all food types cooked or heated in a MWOhousehold were never prepared in a MWO by any of the 96 households. MWO's supplemented but did not replace conventional ranges and other cooking devices.

Manufacturers and MWO cookbooks have emphasized the wide variety of foods that can be acceptably prepared in MWO's, but actual usage rates in this survey did not approach these potentials.

The results of the survey reported here do not support the hypothesis that MWO owners cook or eat less than nonowners, nor is there evidence of an important difference in food selection between owners and non-owners. The more supportable hypothesis is that food quantities and types are the same for owners and non-owners and, lacking positive evidence to the contrary, that average annual cooking energy 
output requirements are also about the same. Energy input requirements may differ, however, depending on the relative efficiencies and usages of the various cooking devices.

It is emphasized that the data used in this report were collected in 1975 and hence involved MWO's with model years no later than 1975. MWO's have undergone considerable refinement since then and their usage may (or may not) be changing, as well. Replication of the MRCA survey for a later year would be informative on this point. 


\section{REFERENCES}

1. Public Law 94-163, Energy Folicy and Conservation Act, December 22, 1975.

2. Public Law 94-385, Energy Conservation and Production Act, August 14, 1976.

3. Hoel, Paul G., Elementary Statistics, John Wiley \& Sons, Inc., New York, 1966.

4. Department of Energy, Final Energy Conservation Test Procedures; Conventional Ranges, Cooking Tops, Ovens Microwave Ovens; Microwave/Conventional Ranges, Furnaces and Vented and Unvented Home Heating Equipment,

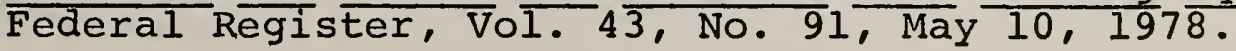


APPENDIX A. DEMOGRAPHIC CHARACTERISTICS AND COMPARISONS OF THE SAMPLED HOUSEHOLDS 
Table Al. Selected characteristics for the U.S. population and the sample of 2000 households used for the Fifth MRCA Menu Census.

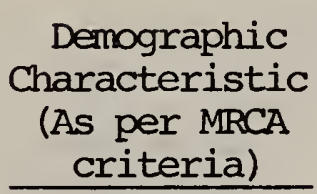

Census Region

Northeast

North Central

South

West

Metro Area Size

Farm

Under 2000

$2500-49999$

$50000-249999$

$250 \quad 000-499999$

$500 \quad 000-999999$

Over 1 million

Household Income

Under $\$ 4000$

$\$ 4000-\$ 6999$

$\$ 7000-\$ 9999$

$\$ 10000-\$ 14999$

$\$ 15000-\$ 24999$

Over $\$ 25000$
Head-of-Household
Education
Under 9 years
9-12 years
over 12 years

Housewife

Employed

Not employed \begin{tabular}{l} 
U.S. Census \\
Households \\
\hline
\end{tabular}

Percent
Fifth Menu

Census Sample

Percent
24.3

24.7

$\begin{array}{ll}27.6 & 27.4\end{array}$

$30.7 \quad 30.3$

$17.4 \quad 17.6$

$4.1 \quad 5.4$

$11.7 \quad 11.0$

$10.8 \quad 11.7$

$\begin{array}{ll}7.8 & 8.0\end{array}$

$10.2 \quad 9.7$

$12.7 \quad 12.1$

$42.7 \quad 42.1$

$17.5 \quad 14.1$

$15.4 \quad 17.1$

$14.6 \quad 15.8$

$22.6 \quad 22.8$

$22.1 \quad 23.7$

$\begin{array}{ll}7.8 & 6.5\end{array}$

23.4

16.7

$48.4 \quad 45.5$

$\begin{array}{ll}48.2 & 37.8\end{array}$

35.0

36.9

$65.0 \quad 63.1$ 
Table Al. Selected characteristics for the U.S. population and the sample of 2000 households used for the Fifth MRCA Menu Census. (continued)

\section{Demographic Characteristic (As per MRCA criteria)}

Household Size

1 person

2 persons

3-4 persons

5 or more persons

Head of Household

Occupation

White collar

Blue collar

Farmer

Not a worker

Number of Children

None

Under 6 years

6-12 years only

13-17 years only

Under $6, \& 6-12$

Under $6, \& 13-17$

6-12, \& 13-17

All 3 ages

Race

White

Non-white

$\begin{aligned} & \text { U.S. Census } \\ & \text { Households }\end{aligned}$
Percent

Fifth Menu

Census Sample

Percent

19.1

18.7

30.8

32.6

17.5

31.4

32.4

17.5

32.5

35.1

38.4

32.0

2.8

2.7

29.6

26.9

55.3

10.7

7.0

8.8

6.8

1.0

8.3

2.1

55.2

9.8

7.4

8.5

8.2

8.2

0.4

1.7

88.7

11.3

91.9

8.1 
Table Al. Selected characteristics for the U.S. population and the sample of 2000 households used for the Fifth MRCA Menu Census. (continued)

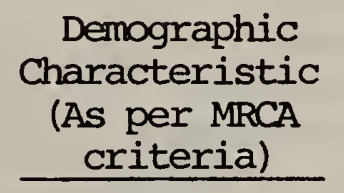

Persons by Age \& Sex

Under 2 Years

Male

Female

2-5 Years

Male

Female

6-12 Years

Male

Female

13-17 Years

Male

Female

18-24 Years

Male

Female

25-44 Years

Male

Female

45-54 Years

Male

Female

55-64 Years

Male

Female

$65 \&$ Older

Male

Female

Total Males

Total Females
U.S. Census

Households

Percent
Fifth Menu

Census Sample

Percent
2.0

1.2

1.9

1.3

3.6

3.4

3.2

6.2

7.3

5.9

6.8

4.9

5.0

4.8

4.9

6.4

6.2

4.2

4.3

12.3

12.4

12.5

13.5

5.2

5.1

5.6

6.3

4.3

3.8

4.9

5.3

4.1

5.8

4.2

7.5

49.0

46.9

51.0

53.1 
Table A2. Comparison of MWO and non-MWO households on the basis of demographic characteristics.

\begin{tabular}{|c|c|c|c|c|c|c|c|c|}
\hline \multirow{3}{*}{$\begin{array}{c}\text { Demographic } \\
\text { Characteristic } \\
\text { (As per MRCA } \\
\text { criteria) }\end{array}$} & \multicolumn{4}{|c|}{$\begin{array}{l}\text { Households Owning } \\
\text { Microwave Ovens }\end{array}$} & \multicolumn{4}{|c|}{$\begin{array}{l}\text { Households Not Owning } \\
\text { Microwave Ovens }\end{array}$} \\
\hline & \multicolumn{2}{|c|}{$\begin{array}{l}\text { Households } \\
\text { in Sample }\end{array}$} & \multicolumn{2}{|c|}{$\begin{array}{l}\text { Persons } \\
\text { in Sample }\end{array}$} & \multicolumn{2}{|c|}{$\begin{array}{l}\text { Households } \\
\text { in Sample }\end{array}$} & \multicolumn{2}{|c|}{$\begin{array}{l}\text { Persons } \\
\text { in Sample }\end{array}$} \\
\hline & Number & $\frac{8}{8}$ & Number & $\frac{\circ}{0}$ & Number & $\%$ & Number & $\frac{0}{8}$ \\
\hline Total Households & 96 & 100.0 & 260 & 100.0 & 96 & 100.0 & 260 & 100.0 \\
\hline \multicolumn{9}{|l|}{ Census Region } \\
\hline Northeast & 15. & 15.6 & 46 & 17.7 & 15 & 15.6 & 46 & 17.7 \\
\hline North Central & 32 & 33.3 & 89 & 34.2 & 32 & 33.3 & 91 & 35.0 \\
\hline South & 29 & 30.2 & 82 & 31.5 & 29 & 30.2 & 78 & 30.0 \\
\hline West & 20 & 20.8 & 43 & 16.5 & 20 & 20.8 & 45 & 17.3 \\
\hline \multicolumn{9}{|l|}{ Metro Area Size } \\
\hline Farm & 3 & 3.1 & 13 & 5.0 & 4 & 4.2 & 14 & 5.4 \\
\hline Under 2500 & 9 & 9.4 & 27 & 10.4 & 7 & 7.3 & 23 & 8.8 \\
\hline $2500-49999$ & 13 & 13.5 & 38 & 14.6 & 13 & 13.5 & 35 & 13.5 \\
\hline $50 \quad 000-249999$ & 4 & 4.2 & 12 & 4.6 & 4 & 4.2 & 12 & 4.6 \\
\hline $250 \quad 000-499999$ & 10 & 10.4 & 28 & 10.8 & 11 & 11.5 & 30 & 11.5 \\
\hline $500 \quad 000-999999$ & 9 & 9.4 & 19 & 7.3 & 10 & 10.4 & 22 & 8.5 \\
\hline Over 1 million & 48 & 50.0 & 123 & 47.3 & 47 & 49.0 & 124 & 47.7 \\
\hline \multicolumn{9}{|l|}{ Household Incame } \\
\hline Under $\$ 4000$ & 10 & 10.4 & 14 & 5.4 & 6 & 6.3 & 8 & 3.1 \\
\hline$\$ 4000-\$ 6999$ & 13 & 13.5 & 25 & 9.6 & 17 & 17.7 & 37 & 14.2 \\
\hline$\$ .7000-\$ 9.999$ & 13 & 13.5 & 39 & 15.0 & 17 & 17.7 & 41 & 15.8 \\
\hline$\$ 10000-\$ 14999$ & 22 & 22.9 & 69 & 26.5 & 23 & 24.0 & 67 & 25.8 \\
\hline$\$ 15000-\$ 24999$ & 30 & 31.3 & 91 & 35.0 & 27 & 28.1 & 84 & 32.3 \\
\hline Over $\$ 25000$ & 8 & 8.3 & 22 & 8.5 & 6 & 6.3 & 23 & 8.8 \\
\hline \multirow{2}{*}{\multicolumn{9}{|c|}{$\begin{array}{l}\text { Head-of-Household } \\
\text { Education }\end{array}$}} \\
\hline & & & & & & & & \\
\hline Under 9 years & 8 & 8.3 & 16 & 6.2 & 13 & 13.5 & 31 & 11.9 \\
\hline $9-12$ years & 43 & 44.8 & 117 & 45.0 & 37 & 38.5 & 97 & 37.3 \\
\hline over 12 years & 45 & 46.9 & 127 & 48.8 & 46 & 47.9 & 132 & 50.8 \\
\hline \multicolumn{9}{|l|}{ Housewife } \\
\hline Employed & 47 & 49.0 & 114 & 43.8 & 47 & 49.0 & 114 & 43.8 \\
\hline Not employed & 49 & 51.0 & 146 & 56.2 & 49 & 51.0 & 146 & 56.2 \\
\hline \multicolumn{9}{|l|}{ Household Size } \\
\hline 1 person & 21 & 21.9 & 21 & 8.1 & 17 & 17.7 & 17 & 6.5 \\
\hline 2 persons & 32 & 33.3 & 64 & 24.6 & 33 & 34.4 & 66 & 25.4 \\
\hline 3-4 persons & 32 & 33.3 & 114 & 43.8 & 37 & 38.5 & 129 & 49.6 \\
\hline 5 or more persons & 11 & 11.5 & 61 & 23.5 & 9 & 9.4 & 48 & 18.5 \\
\hline
\end{tabular}


Table A2. Comparison of MWO and non-MWO households on the basis of demographic characteristics. (continued)

\begin{tabular}{|c|c|c|c|c|c|c|c|c|}
\hline \multirow{3}{*}{$\begin{array}{c}\text { Demographic } \\
\text { Characteristic } \\
\text { (As per MRCA } \\
\text { Criteria) }\end{array}$} & \multicolumn{4}{|c|}{$\begin{array}{l}\text { Households Owning } \\
\text { Microwave Ovens }\end{array}$} & \multicolumn{4}{|c|}{$\begin{array}{l}\text { Households Not Owning } \\
\text { Microwave Ovens }\end{array}$} \\
\hline & \multicolumn{2}{|c|}{$\begin{array}{l}\text { Households } \\
\text { in Sample }\end{array}$} & \multicolumn{2}{|c|}{$\begin{array}{l}\text { Persons } \\
\text { in Sammle }\end{array}$} & \multicolumn{2}{|c|}{$\begin{array}{l}\text { Households } \\
\text { in Sample }\end{array}$} & \multicolumn{2}{|c|}{$\begin{array}{l}\text { Persons } \\
\text { in Sample }\end{array}$} \\
\hline & Number & $\frac{8}{8}$ & Number & $\%$ & Number & $\%$ & Number & $\frac{0}{0}$ \\
\hline \multicolumn{9}{|l|}{$\begin{array}{l}\text { Head of Household } \\
\text { Occupation }\end{array}$} \\
\hline White collar & 47 & 49.0 & 126 & 48.5 & 45 & 46.9 & 124 & 47.7 \\
\hline Blue collar & 29 & 30.2 & 98 & 37.7 & 31 & 32.3 & 98 & 37.7 \\
\hline Farmer & - & -- & -- & - & 2 & 2.1 & 8 & 3.1 \\
\hline Not a worker & 20 & 20.8 & 36 & 13.8 & 18 & 18.8 & 30 & 11.5 \\
\hline \multicolumn{9}{|l|}{ Number of Children } \\
\hline None & 57 & 59.4 & 98 & 37.7 & 56 & 58.3 & 101 & 38.8 \\
\hline Under 6 years & 8 & 8.3 & 29 & 11.2 & 14 & 14.6 & 48 & 18.5 \\
\hline 6-12 years only & 8 & 8.3 & 36 & 13.8 & 5 & 5.2 & 18 & 6.9 \\
\hline $13-17$ years only & 11 & 11.5 & 39 & 15.0 & 7 & 7.3 & 26 & 10.0 \\
\hline Under $6, \& 6-12$ & 6 & 6.3 & 29 & 11.2 & 10 & 10.4 & 45 & 17.3 \\
\hline Under $6, \& 13-17$ & - & $=$ & -- & - & - & -- & - & - \\
\hline $6-12, \& 13-17$ & 5 & 5.2 & 23 & 8.8 & 4 & 4.2 & 22 & 8.5 \\
\hline All 3 ages & 1 & 1.0 & 6 & 2.3 & - & - & -- & - \\
\hline \multicolumn{9}{|l|}{ Race } \\
\hline White & 90 & 93.8 & 244 & 93.8 & 90 & 93.8 & 243 & 93.5 \\
\hline Non-white & 6 & & 16 & & & 6.3 & 17 & 6.5 \\
\hline \multirow{2}{*}{\multicolumn{9}{|c|}{ Season of Diary }} \\
\hline & & & & & & & & \\
\hline 1975 & 27 & 28.1 & 70 & 26.9 & 24 & 25.0 & 73 & 28.1 \\
\hline Apr- & 24 & 25.0 & 71 & 27.3 & 26 & 27.1 & 65 & 25.0 \\
\hline Jul-Sep 1975 & 26 & 27.1 & 66 & 25.4 & 17 & 17.7 & 41 & 15.8 \\
\hline Oct-Dec 1975 & 19 & 19.8 & 53 & 20.4 & 29 & 30.2 & 81 & 31.2 \\
\hline \multicolumn{9}{|l|}{ Religion } \\
\hline Protestant & 36 & 37.5 & 88 & 33.8 & 32 & 33.3 & 89 & 34.2 \\
\hline Catholic & 11 & 11.5 & 36 & 13.8 & 13 & 13.5 & 39 & 15.0 \\
\hline Jewish & 4 & 4.2 & 17 & 6.5 & 2 & 2.1 & 6 & 2.3 \\
\hline Other & -- & - & -- & - & 2 & 2.1 & 3 & 1.2 \\
\hline None & -- & - & - & - & 1 & 1.0 & 1 & 0.4 \\
\hline \multicolumn{9}{|l|}{ Economic Class } \\
\hline High & 25 & 26.0 & 79 & 30.4 & 24 & 25.0 & 81 & 31.2 \\
\hline Upper middle & 30 & 31.3 & 91 & 35.0 & 21 & 21.9 & 60 & 23.1 \\
\hline Lower middle & 16 & 16.7 & 45 & 17.3 & 28 & 29.2 & 74 & 28.5 \\
\hline Low & 25 & 26.0 & 45 & 17.3 & 23 & 24.0 & 45 & 17.3 \\
\hline \multicolumn{9}{|l|}{ Household Head } \\
\hline Male, spouse present & 70 & 72.9 & 226 & 86.9 & 72 & 75.0 & 221 & 85.0 \\
\hline Male, no & 1 & 1.0 & $\frac{1}{2}$ & 0.4 & $\overline{-\overline{2}}$ & $\overline{5}$ & $\overline{30}$ & 150 \\
\hline Fema & 25 & 26.9 & 33 & 12.7 & 24 & 25.0 & 39 & 15.0 \\
\hline
\end{tabular}


Table A2. Comparison of MWO and non-MWO households on the basis of demographic characteristics. (continued)

\begin{tabular}{|c|c|c|c|c|c|c|c|c|}
\hline \multirow{3}{*}{$\begin{array}{c}\text { Demographic } \\
\text { Characteristic } \\
\text { (As per MRCA } \\
\text { criteria) }\end{array}$} & \multicolumn{4}{|c|}{$\begin{array}{l}\text { Households Owning } \\
\text { Microwave Ovens }\end{array}$} & \multicolumn{4}{|c|}{$\begin{array}{l}\text { Households Not Owning } \\
\text { Microwave Ovens }\end{array}$} \\
\hline & \multicolumn{2}{|c|}{$\begin{array}{l}\text { Households } \\
\text { in Sample }\end{array}$} & \multicolumn{2}{|c|}{$\begin{array}{l}\text { Persons } \\
\text { in Sample }\end{array}$} & \multicolumn{2}{|c|}{$\begin{array}{l}\text { Households } \\
\text { in Sample }\end{array}$} & \multicolumn{2}{|c|}{$\begin{array}{l}\text { Persons } \\
\text { in Sample }\end{array}$} \\
\hline & Number & $\frac{9}{8}$ & Number & $\%$ & Number & $\frac{9}{8}$ & Number & $\%$ \\
\hline \multicolumn{9}{|c|}{ Persons by Age \& Sex } \\
\hline Under 2 Years & & & & & & & & \\
\hline Male & 2 & 2.1 & 2 & 0.8 & 3 & 3.1 & 3 & 1.2 \\
\hline Female & 3 & 3.1 & 3 & 1.2 & 6 & 6.3 & 6 & 2.3 \\
\hline 2-5 Years & & & & & & & & \\
\hline Male & 9 & 9.4 & 9 & 3.5 & 11 & 11.5 & 13 & 5.0 \\
\hline Female & 7 & 7.3 & 7 & 2.7 & 11 & 11.5 & 11 & 4.2 \\
\hline 6-12 Years & & & & & & & & \\
\hline Male & 15 & 15.6 & 21 & 8.1 & 12 & 12.5 & 12 & 4.6 \\
\hline Female & 14 & 14.6 & 17 & 6.5 & 12 & 12.5 & 15 & 5.8 \\
\hline 13-17 Years & & & & & & & & \\
\hline Male & 8 & 8.3 & 8 & 3.1 & 5 & 5.2 & 5 & 1.9 \\
\hline Female & 15 & 15.6 & 16 & 6.2 & 11 & 11.5 & 16 & 6.2 \\
\hline 18-24 Years & & & & & & & & \\
\hline Male & 11 & 11.5 & 11 & 4.2 & 7 & 7.3 & 7 & 2.7 \\
\hline Female & 11 & 11.5 & 11 & 4.2 & 7 & 7.3 & 7 & 2.7 \\
\hline 25-44 Years & & & & & & & & \\
\hline Male & 38 & 39.6 & 38 & 14.6 & 41 & 42.7 & 41 & 15.8 \\
\hline Female & 40 & 41.7 & 40 & 15.4 & 47 & 49.0 & 48 & 18.5 \\
\hline 45-54 Years & & & & & & & & \\
\hline Male & 10. & 10.4 & 10 & 3.8 & 9 & 9.4 & 9 & 3.5 \\
\hline Female & 18 & 18.8 & 18 & 6.9 & 15 & 15.6 & 15 & 5.8 \\
\hline 55-64 Years & & & & & & & & \\
\hline Male & 11 & 11.5 & 11 & 4.2 & 12 & 12.5 & 12 & $\begin{array}{l}4.6 \\
5.4\end{array}$ \\
\hline Female & 14 & 14.6 & 14 & 5.4 & 14 & 14.6 & 14 & 5.4 \\
\hline $65 \&$ Older & & & & 35 & & & & \\
\hline $\begin{array}{l}\text { Male } \\
\text { Female }\end{array}$ & $\begin{array}{r}9 \\
15\end{array}$ & $\begin{array}{r}9.4 \\
15.6\end{array}$ & $\begin{array}{r}9 \\
15\end{array}$ & $\begin{array}{l}3.5 \\
5.8\end{array}$ & $\begin{array}{l}10 \\
16\end{array}$ & $\begin{array}{l}10.4 \\
18.7\end{array}$ & $\begin{array}{l}10 \\
16\end{array}$ & $\begin{array}{l}3.8 \\
6.2\end{array}$ \\
\hline Total Males & 75 & 78.1 & 119 & 45.8 & 77 & 80.2 & 112 & 43.1 \\
\hline Total Females & 95 & 99.0 & 141 & 54.2 & 96 & 100.0 & 148 & 56.9 \\
\hline
\end{tabular}


Table A2. Comparison of MWO and non-MWO households on the basis of demographic characteristics. (continued)

\begin{tabular}{|c|c|c|c|c|c|c|c|c|}
\hline \multirow{3}{*}{$\begin{array}{c}\text { Demographic } \\
\text { Characteristic } \\
\text { (As per MRCA } \\
\text { criteria) } \\
\end{array}$} & \multicolumn{4}{|c|}{$\begin{array}{l}\text { Households Owning } \\
\text { Microwave Ovens }\end{array}$} & \multicolumn{4}{|c|}{$\begin{array}{l}\text { Households Not Owning } \\
\text { Microwave Ovens }\end{array}$} \\
\hline & \multicolumn{2}{|c|}{$\begin{array}{l}\text { Households } \\
\text { in Sample }\end{array}$} & \multicolumn{2}{|c|}{$\begin{array}{l}\text { Persons } \\
\text { in Sample }\end{array}$} & \multicolumn{2}{|c|}{$\begin{array}{l}\text { Households } \\
\text { in Sample }\end{array}$} & \multicolumn{2}{|c|}{$\begin{array}{l}\text { Persons } \\
\text { in Sample }\end{array}$} \\
\hline & Number & $\frac{9}{8}$ & Number & $\frac{0}{0}$ & Number & $\div$ & Number & $\frac{\%}{6}$ \\
\hline \multicolumn{9}{|l|}{$\begin{array}{l}\text { Persons on Diet by } \\
\text { Age \& Sex }\end{array}$} \\
\hline \multicolumn{9}{|l|}{$\begin{array}{l}\text { Under } 18 \text { Years } \\
\text { On diet }\end{array}$} \\
\hline Male & - & -- & -- & -- & -- & -- & -- & -- \\
\hline Female & 9 & 9.4 & 9 & 3.5 & 7 & 7.3 & 9 & 3.5 \\
\hline Male & 30 & 31.3 & 40 & 15.4 & 24 & 25.0 & 33 & 12.7 \\
\hline Female & 28 & 29.2 & 34 & 13.1 & 26 & 27.1 & 39 & 15.0 \\
\hline \multicolumn{9}{|l|}{18 \& Over Losing } \\
\hline Male & 12 & 12.5 & 12 & 4.6 & 12 & 12.5 & 13 & 5.0 \\
\hline Female & 30 & 31.3 & 31 & 11.9 & 23 & 34.4 & 34 & 13.1 \\
\hline \multicolumn{9}{|l|}{$18 \&$ Over Watching } \\
\hline Male & 3 & 3.1 & 4 & 1.5 & 1 & 1.0 & 2 & 0.8 \\
\hline Female & 6 & 6.3 & 6 & 2.3 & 9 & 9.4 & 9 & 3.5 \\
\hline \multirow{2}{*}{\multicolumn{9}{|c|}{18 \& Over Gaining }} \\
\hline Male & & 1.0 & 1 & 0.4 & - & -- & - & -- \\
\hline Female & - & - & - & -- & 1 & 1.0 & 1 & 0.4 \\
\hline \multicolumn{9}{|l|}{$\begin{array}{l}18 \text { \& Over Medical/ } \\
\text { Health }\end{array}$} \\
\hline Male & 11 & 11.5 & 11 & 4.2 & 7 & 7.3 & 7 & 2.7 \\
\hline Female & 13 & 13.5 & 13 & 5.0 & 11 & 11.5 & 11 & 4.2 \\
\hline \multicolumn{9}{|l|}{18 \& Over None } \\
\hline Male & 50 & 52.1 & 51 & 19.6 & 55 & 57.3 & 57 & 21.9 \\
\hline Female & 48 & 50.0 & 48 & 18.5 & 45 & 46.9 & 45 & 17.3 \\
\hline \multicolumn{9}{|l|}{ Total } \\
\hline Male & 75 & 78.1 & 119 & 45.8 & 77 & 80.2 & 112 & 43.1 \\
\hline Female & 95 & 99.0 & 141 & 54.2 & 96 & 100.0 & 148 & 56.9 \\
\hline
\end{tabular}


Contingency tables are arrays of numbers that subdivide some total number of events into cells that indicate the observed numbers of events, say Servings or Eatings, having combinations of properties or attributes. These properties range over all possibilities within each classification system. For example, MWO-owners and non-owners would define all possibilities with respect to the MWO ownership attribute. Household size and food type are examples of other attribute systems useful in categorizing the same events. The analyses in this report are limited to "twoway" tables, meaning that the events are allocated according to only two attribute systems in each case. Higher order tables (three-way, four-way, etc.) are possible, with the analysis principles remaining the same.

Contingency analysis is a formal method to test for the presence or absence of association (not causation) among the attribute systems. The findings provide guidance on the rejection or acceptance of the "null hypothesis," $\mathrm{H}_{\mathrm{O}}$, meaning that the attribute systems do, or do not, display association. For example, do owners eat disproportionately more leftovers than non-owners? The data in the following table were taken from Table 1.

\begin{tabular}{|c|c|c|c|}
\hline Eatings & $\begin{array}{l}\text { MwO } \\
\text { Owners }\end{array}$ & $\begin{array}{c}\text { Non- } \\
\text { Owners }\end{array}$ & $\begin{array}{c}\text { Row } \\
\text { Totals }\end{array}$ \\
\hline First Time & $\begin{array}{rl}15 & 507 \\
(15 & 501)\end{array}$ & $\begin{array}{ll}15 & 623 \\
(15 & 629)\end{array}$ & $\left.\begin{array}{rl}31 & 130 \\
(31 & 130\end{array}\right)$ \\
\hline Leftovers & $\left.\begin{array}{ll}1 & 508 \\
(1 & 514\end{array}\right)$ & $\begin{array}{ll}1 & 532 \\
(1 & 526)\end{array}$ & $\left.\begin{array}{cc}3 & 040 \\
(3 & 040\end{array}\right)$ \\
\hline Column Totals & $\begin{array}{cl}17 & 015 \\
(17 & 015)\end{array}$ & $\begin{array}{ll}17 & 155 \\
(17 & 155)\end{array}$ & $\left.\begin{array}{rl}34 & 170 \\
(34 & 170\end{array}\right)$ \\
\hline
\end{tabular}

$\chi^{2}=0.048, p \simeq 83 \%, 1$ degree of freedom (df)

( $x^{2}$ calculated without rounding of expected values)

The upper numbers in each pair in the table are the actual observations. The lower numbers, in parentheses, are the values that would be "expected" if one only knew the row and column totals and assumed that there was no association between the two attribute systems. The chi-square $\left(x^{2}\right)$ statistic is determined by 


$$
x^{2}=\sum_{\substack{\text { Row }_{i} \text { Columns } \\(i, j)}} \frac{\text { Expected } \left._{i, j}-\text { Observed }_{i, j}\right)^{2}}{\text { Expected }_{i, j}}=0.048 .
$$

The number of degrees of freedom (df) is the number of values in the table that could be varied independently, while holding the row and column totals fixed. Not counting these totals, only one of the other values may be varied in this sense, and having chosen a value, the other entries are determined. Hence, $\mathrm{df}=1$.

Reference to a table of $x^{2}$ statistics shows that differences between expected and observed results at least as large as this could have occurred roughly 83 percent of the times such an experiment was run, when there is really no difference in the populations from which these samples originated. While the investigator must decide for himself, based on the consequences of being wrong, it appears advisable in this case to accept the null hypothesis--there is no association between attributes under consideration. That is, there is no statistically supportable difference between owners and non-owners with respect to the proportions of first time and leftover eatings. 
APPENDIX C. HOUSEHOLD AND MEAL CHARACTERISTIC PROFILES FOR MWO-OWNERS AND NON-OWNERS 
FIFTH NATIONAL HOUSEHOLD MENU CENSUS

JANUARY THRU DECEMBER 1975

-- ANNUAL SUMMARY --

REPORT TO

NATIONAL BUREAU OF STANDARDS *

$\underline{\text { INDEX }}$

REPORT - A - FOODS

Description of User Groups

Report A - OWNER - IN MWO - ALL FOODS

- LEFTOVERS

- LIQUIDS

- ANIMAL PROTEIN

- FRUITS \& VEGS.

- BAKED GOODS

- HOT DISHES

- RAW/SCRATCH

- CONVENIENCE FDS.

- FROZEN FOODS

** Report A - OWNER - CONV. OVEN - ALL FOODS

- LEFTOVERS

- LIQUIDS

- ANIMAL PROTEIN

- FRUITS \& VEGS.

- BAKED GOODS

- HOT DISHES

- RAW/SCRATCH

- CONVENIENCE FOODS

- FROZEN FOODS

** Report A - NON OWNER - CONV. OVEN - ALL FOODS

- LEFTOVERS

- LIQUIDS

- ANIMAL PROTEIN

- FRUITS \& VEGS.

- BAKED GOODS

- HOT DISHES

- RAW/SCRATCH

- CONVENIENCE FDS.

- FROZEN FOODS
Report I.D. No.

$$
\begin{array}{r}
7303-01 \\
-02 \\
-03 \\
-04 \\
-05 \\
-06 \\
-07 \\
-08 \\
-09 \\
-10
\end{array}
$$

$7303-11$

$-12$

$-13$

$-14$

$-15$

$-16$

$-17$

$-18$

$-19$

$-20$

$7303-21$

$-22$

- 23

- 24

- 25

- 26

- 22

- 28

- 29

- 30

*Data summaries only for report I.D. numbers 7303-01, -11 , and $-2 i$ are included in this report.

**"Conv. Oven" should read "Not in MWO." 
Headnotes for Tables $\mathrm{C}-1, \mathrm{C}-2, \mathrm{C}-3$ and $\mathrm{C}-4$.

Index Times Served $=100 \times \frac{\text { Total HH }}{\text { Total Servings }} \times \frac{\text { Serving }}{\mathrm{HH}^{\prime} \mathrm{S} \text { Serving }}$

Index No. of Eatings $=100 \times \frac{\text { Total HH }}{\text { Total Eatings }} \times \frac{\text { Eatings }}{\mathrm{HH} \text { Serving }}$

or, where appropriate,

$$
=100 \times \frac{\text { Total Eaters }}{\text { Total Eatings }} \times \frac{\text { Eatings }}{\text { Eater Sample Size }}
$$

C.M. = Carried Meals (e.g., bag lunches prepared at home) $\mathrm{DK}=$ Don' $t$ know 


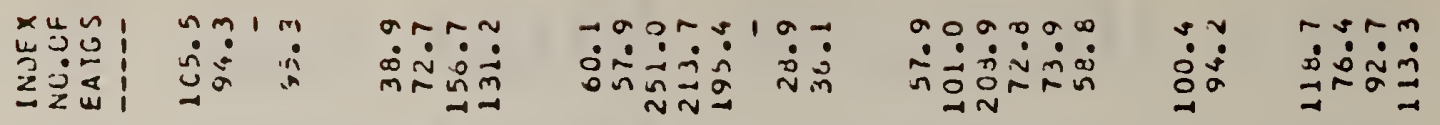

岩焉|

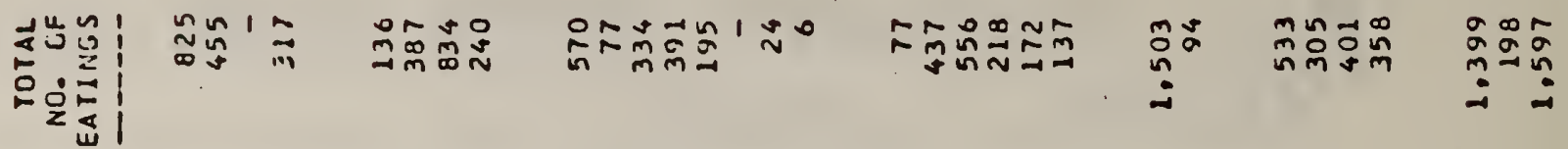

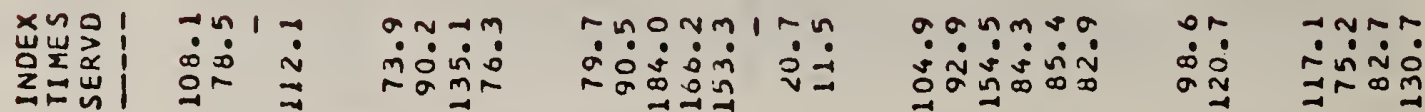

战崖|

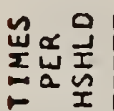

$+\infty 1: 0$

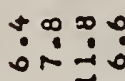

9:0ำ100

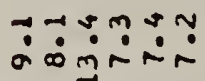

20011

出虽! $\sim 21 \backsim$

ขึ

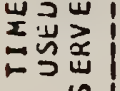

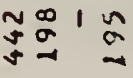

กำกำ

nmosolon

ำํำำำำ

कृ

- $\simeq$ อำ

H

os

$4+4$

(1)

$\cup U$

(

บ i

is

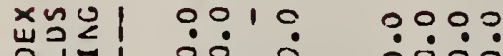

영 :

00000100

0.0090

是至出

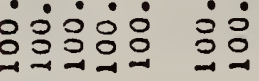

웅용ㅇㅇ

:

$0: 00$

กnก⿻

$\check{\sim} \dot{\sim} \infty$

-nul ON

amm

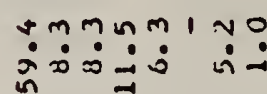

$\because \because \because \infty \bullet$

$\simeq \infty$

$\operatorname{nin}_{n \rightarrow \infty}^{\infty}$

$\operatorname{onn} n 1$ m이

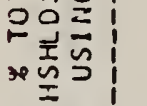

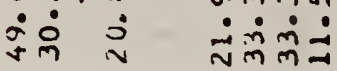

过这!

0010

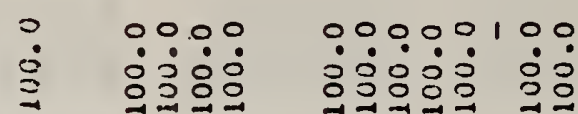

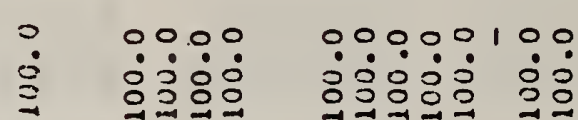

出地

อ이

₹

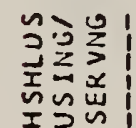

$\vec{N} N \tilde{M}=$

$n^{\infty} \infty \neq 01$ in $\rightarrow$

ㅇํㅇㅇำ

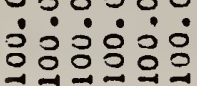

웅

$\infty \stackrel{\sim}{0} \underset{\sim}{\infty} \pm$

30

$\ddot{m}: \dot{0}$

어어은

도

प2 4

동

$\begin{array}{ll}4 & \text { vै } \\ 0 & 0 \\ 1 & 0 \\ 3 & 0\end{array}$

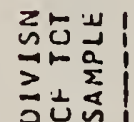

문 जmm

i.

แूนุำ

ป⿻ 1 은

Nmmin

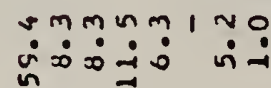

$m \rightarrow \div \infty$

in

$\because 0 \div 0$

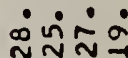

00011 ㅇํㅇㅇㅡ

굴

ลีM

$\infty \stackrel{N}{\infty} \div \pm \pm$ 号芒落

๙

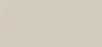

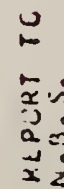
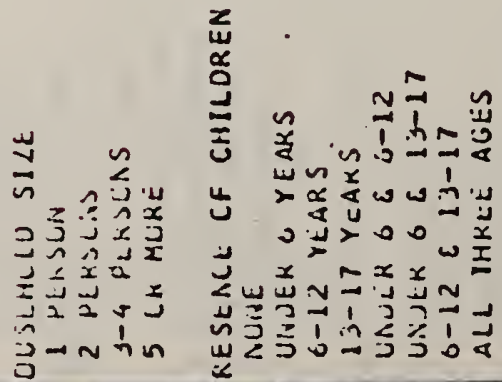

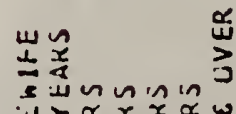

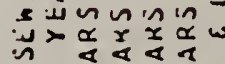

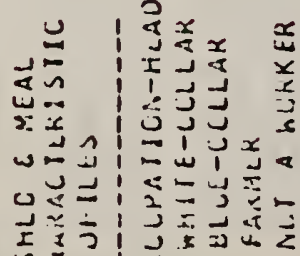

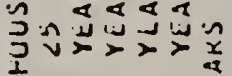

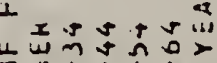

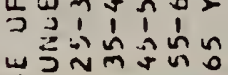

$00 \% 0$

11111

웅응ㅇㅇ

กั $\stackrel{\sim}{\sim}$

$\stackrel{n}{n} \approx 011$

$\because 0 \rightarrow \infty$

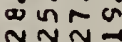

กับำ

111111 
㫨通

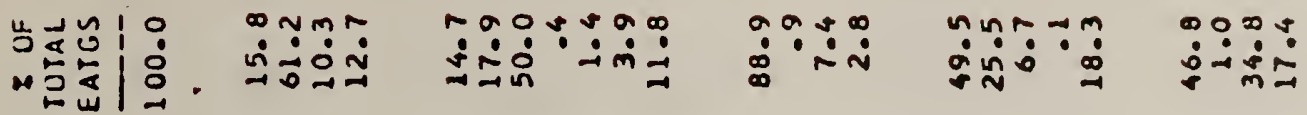

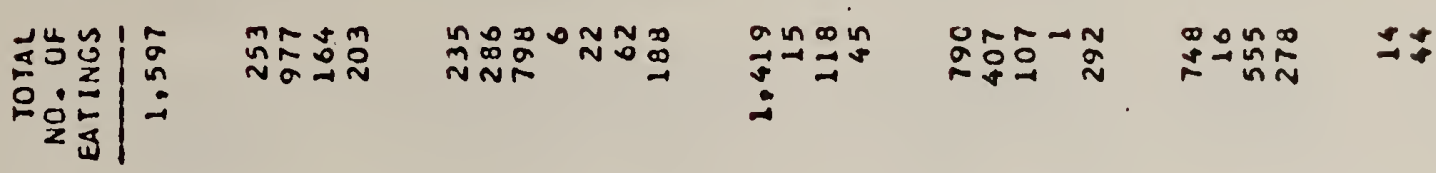

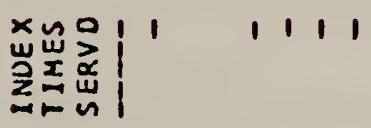

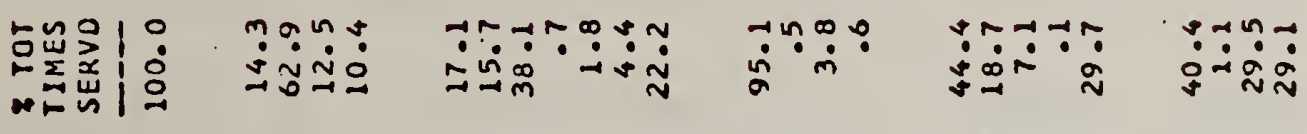

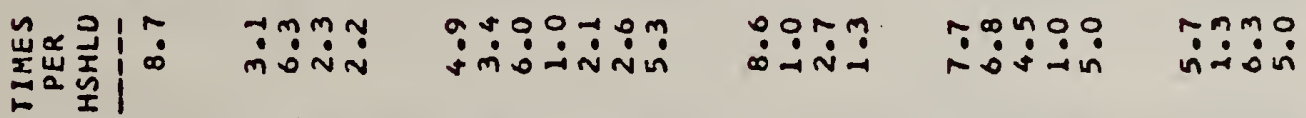

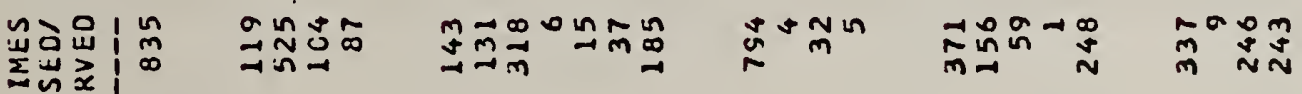

\section{崌虽导|}

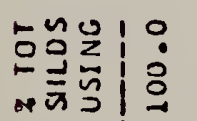

$\operatorname{lin} 0$

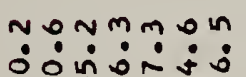

$\min \underset{\min }{\min }$

11111

1111

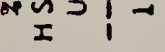

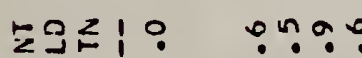

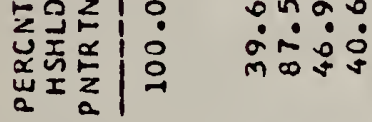

ㅇำ

คู่

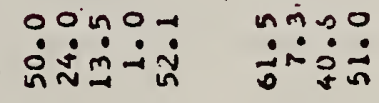

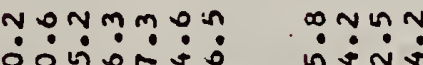

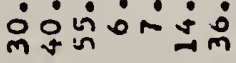

nूं

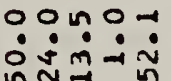

nimo?

น.

主盗这

苩艺岕!

岕



究骂出| 1111

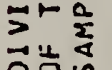

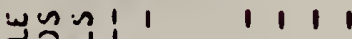

至通

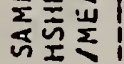
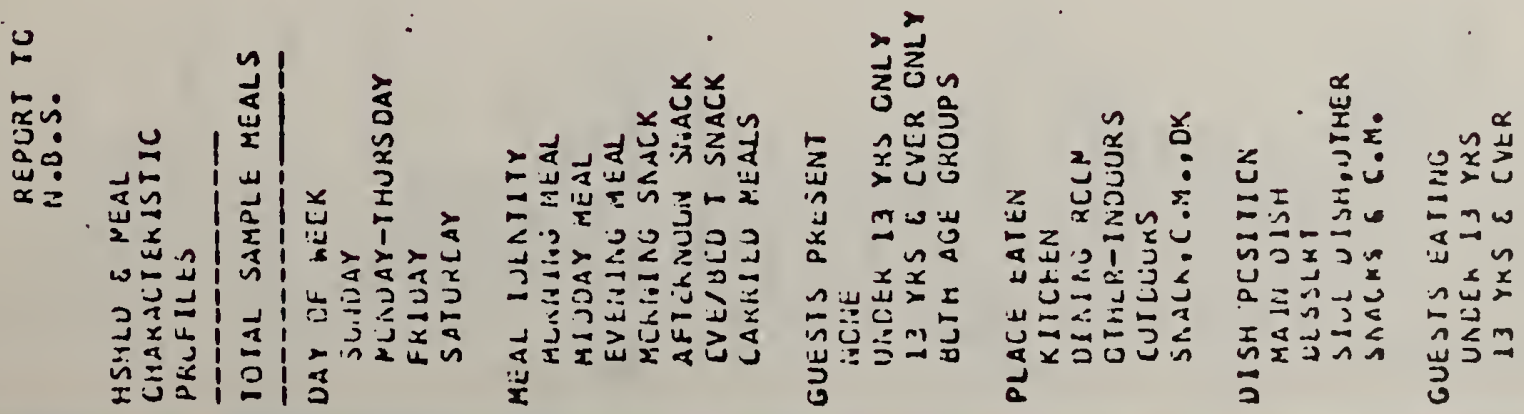
0
0
0
0
$4-1$
1
0
4

0.

ช

$-10$

थ

H

05

00

$\sigma$

6

ن i

元苍

岸

o.

है

$0+$

-1

돌

is

30

도

t4

(0)

క

-

u

1) a

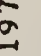

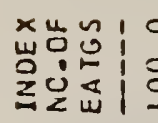

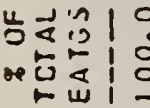

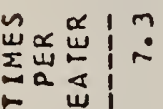

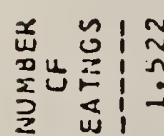

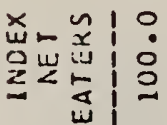

5 ร 10 此范皆

艺茫吉!

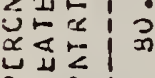
a $=1$

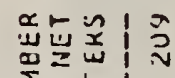
站出尔

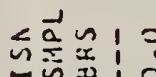

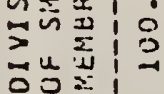

மே

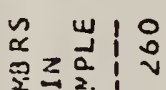

से

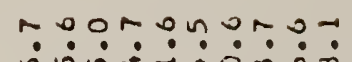

กัก

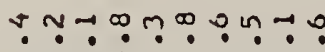

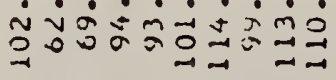

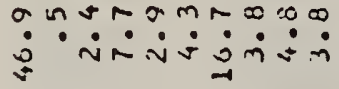

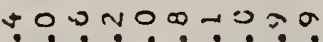

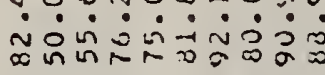

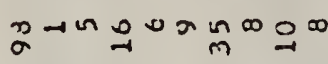

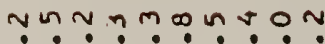

เท் $\dot{\sim} \dot{\sim} \dot{\sim} \dot{i} \dot{\sim} \dot{\sim}$

$\rightarrow \rightarrow-a n \pi$ G

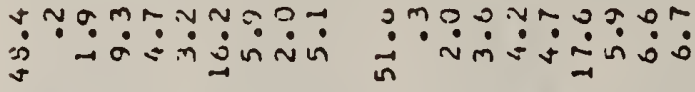

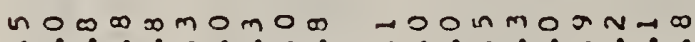

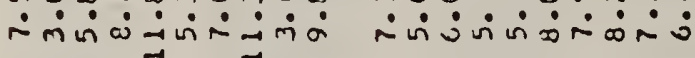

im

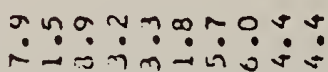

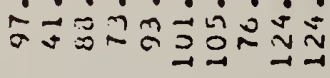

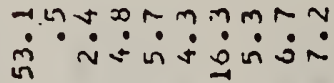

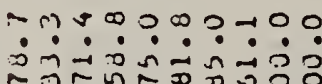

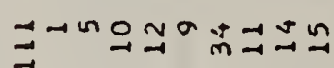

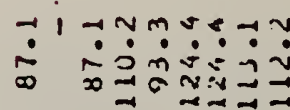

0

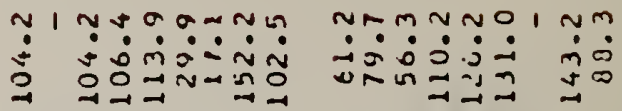

$010 m \eta n \pi t \rightarrow \rightarrow \infty 5 n 001$ nm

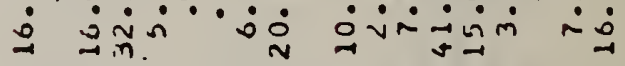

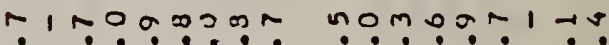

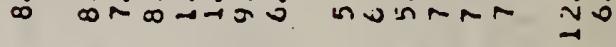

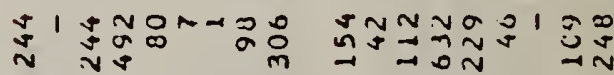

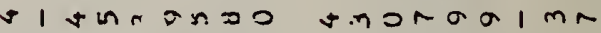

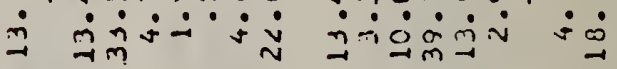

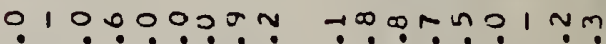

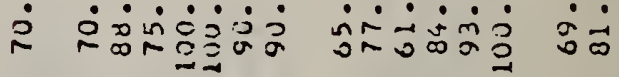

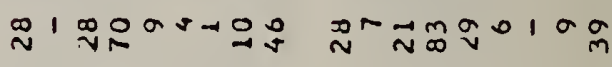
a

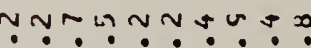

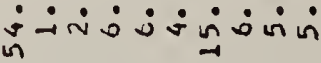

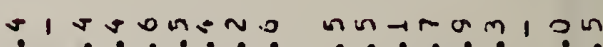

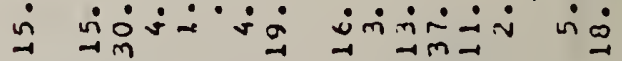
अ

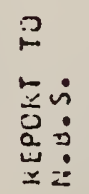

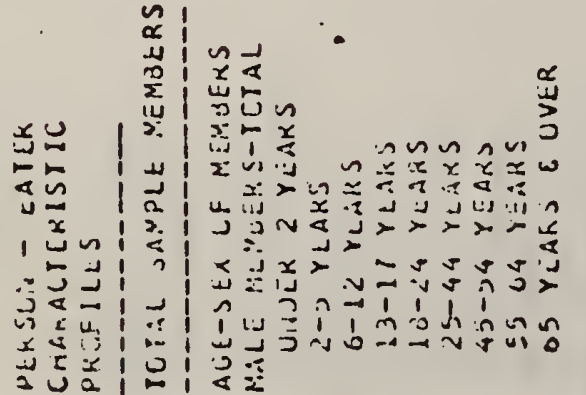

b

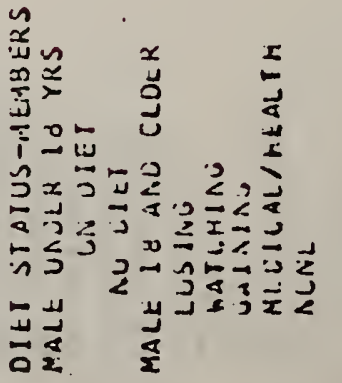

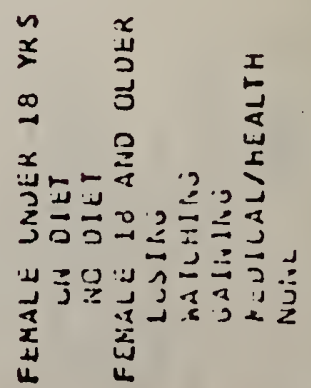


$\begin{array}{ll:l}* u & \sim & 0\end{array}$ $\because 0 \stackrel{1}{2}$ ?

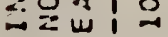

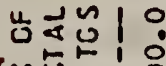

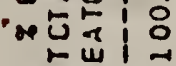

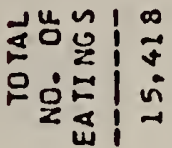

n

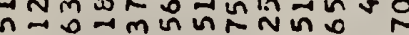
Nंñ்⿻一 mora.tor $\dot{0} \dot{\sim} \dot{0} \dot{0} \dot{0}$ $\rightarrow-1 \rightarrow$

o r. nana niminimin

$+\infty \sin c h$

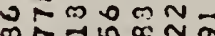
an $\rightarrow-1$ $\ddot{\theta} \dot{i} \dot{*} \dot{m}$

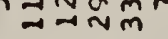

$\sim 0 N N R$ $\overrightarrow{0}+\vec{m} m m$

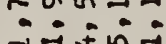

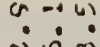

c) 0 a

anc
$N \leqslant \infty N m \infty m m \infty n+m$

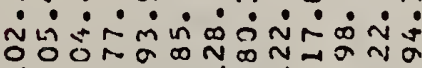
永出出:

노ำำ 路出| 宕出呈|:

出定! 量 河出!

$N N+-00 N N m m+N o$

$r \rightarrow 0 r-m$ nomanor $\therefore 0$ i 윴

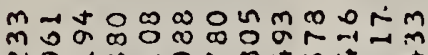

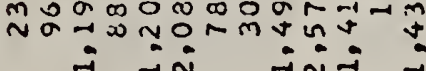
总欪离: mí $\dot{0} \dot{0} \dot{0} \dot{0} \dot{0} \dot{0} \dot{0} \dot{0}$

$$
\text { (1) }
$$

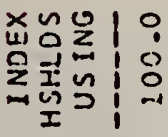

เ幺ับ!

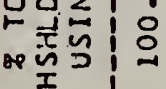

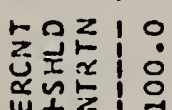

\section{a}

นิ이유

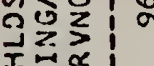

苛出出

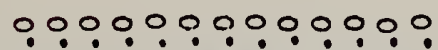

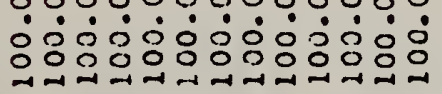

H Un ORMMNTNMOD

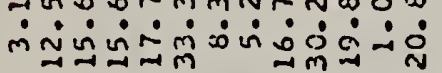

0000000:0000\%

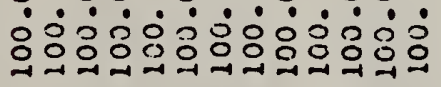

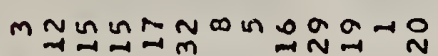

岹 $x \supset \backsim$

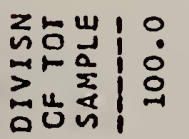

แู่ษ

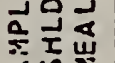

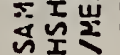

m n

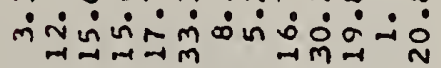

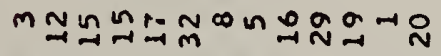

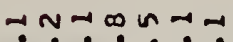
$\dot{\infty} \dot{0} \dot{0} \dot{0} \dot{0}$ $N=\infty$ w $0=10$

ormนtm.0

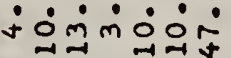

$m \infty$ in $m \cup \sim m$

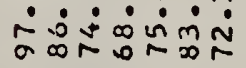

$N-1 \sigma m \sim m a$ NัẵN?

0.00000

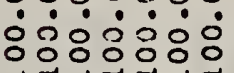

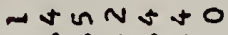
$\dot{m} \dot{m} \dot{\sim} \dot{\sim} \dot{\sigma} \dot{\rho}$

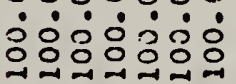

$m a m+0 a x$
บนกษดน ஸें大்

aนกง $\dot{\omega} \ddot{\sim} \dot{\sim} \dot{M}$

?.0.?

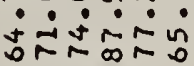

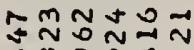

ธัง in

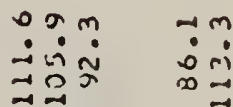

$m$ in $N$ บ

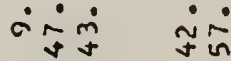

$\infty$ in

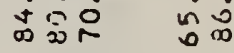

กํำ

क.t.

mi mi
0000000

웅ㅇㅇㅇ

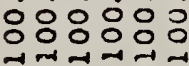

0.0 웅

$\tan \Omega m m$ $\dot{0} \dot{m} \dot{\sim} \dot{\sim} \dot{m}$

$m \infty \sigma$ ?.

000000 용ㅇㅇ

000 웅

엄프늠

$\infty m$ in

$\tan$

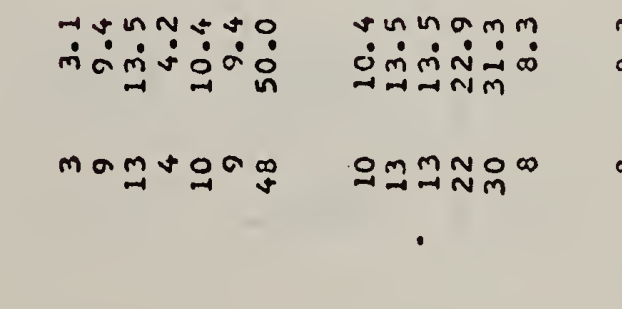

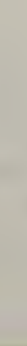

o

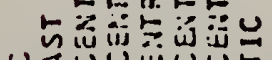

要离

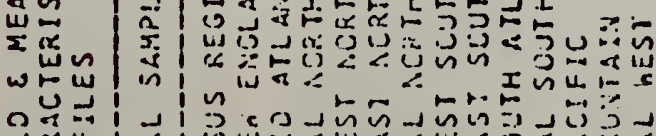

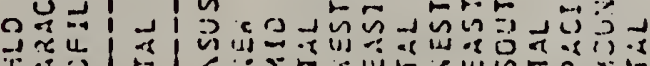

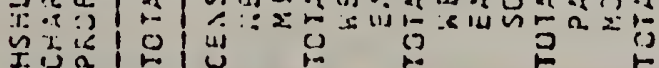

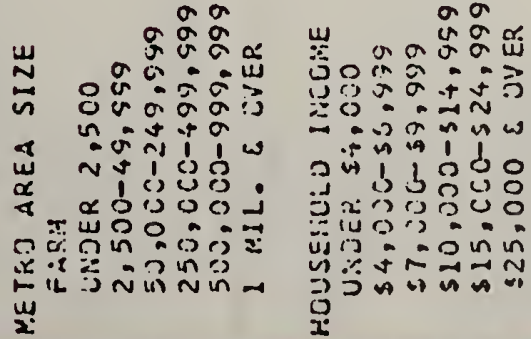

00

웅

$\circ 0$ $\stackrel{a}{a}$

00 웅 a 


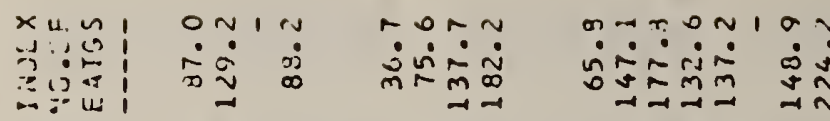

$m \neq 00$ in 0

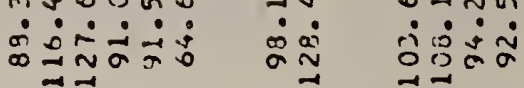

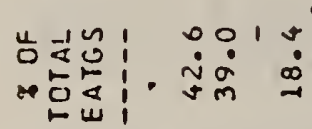

o Na\%

$\because m e n ? 10 m$

ช

$\dot{m} \dot{\sim} \dot{\sim} \dot{\sim} \dot{\sim}$

เ $m \rightarrow m s$

$\because \dot{m} \div \dot{m} \dot{0}$

$\underset{0}{0}$

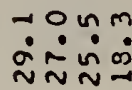

$\because 20111$

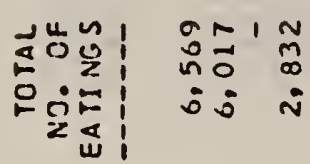

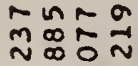

imin

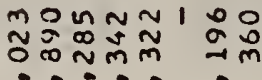

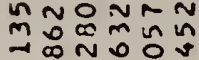

imin:

$\underset{-1}{+\infty}$

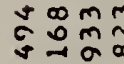

अंकि

ㅇำ

0
O
0
$4-1$

눙

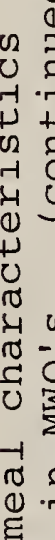

究

ס

$\rightarrow$ 요

(1)

ט 10

采

c

(1)

द्व

(1)

- 낭

1 0

U

$\begin{array}{ll}0 & 0 ? \\ 0 & 0 \\ 0 & 01 \\ 0 & 01\end{array}$

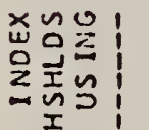

$\begin{array}{lll}0 & 1 & 0 \\ 0 & 0 & 0 \\ 0 & 0 & 0\end{array}$

5 nO! ON

인

$\therefore \dot{0}$

过正

乐垔々

$\stackrel{n}{a}$

a

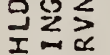

Iป็น

0010

용 웅

$000 \%$

ㅇㅇㅇㅇㅇㅇ

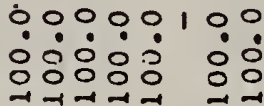

$000.90 \%$

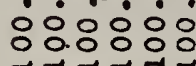

जmmn जिmm

$m \rightarrow \sim \infty ?$

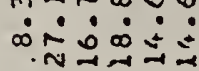

00000100

0000

응응ㅇㅇ음

กัก

Nก๊ักี
눈르

>r玄 कิ

แู้น

a일

品 至崖
ONIa ammin i̊ $\quad \dot{N}$ तimma

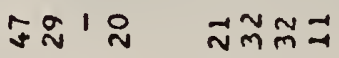

형요용

$n^{\infty} \infty 01 \mathrm{in}-$
$000 \% 00$

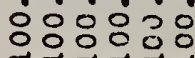

$\infty \infty 0 \infty+5$ $\div m m n m 1 \sim 0$ in

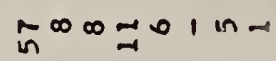
$\dot{\infty} \dot{N} \dot{0} \dot{0}: \dot{4}$ $\infty \stackrel{\sim}{\infty} \underset{\sim}{\infty}$ $m \because \div \infty \because 0$
$0: 0$

80

$\infty m$

in:

$0 \%$

웅

우
$0: 0 \%$

응ㅇㅇㅇㅜ

$\rightarrow 0-\infty$

$\therefore$ iñ

$\sim N N$

0000

영ㅇㅇㅇ

$n+\infty$

ผ :m? 111 요용

1,1111 유요 11

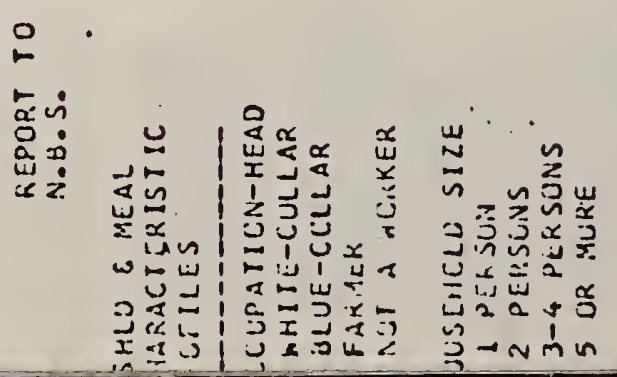

孞

4

峁! $\stackrel{\infty}{m} \dot{m}$

$\rightarrow 0 \rightarrow \infty$ $\stackrel{\infty}{\sim} \sim \dot{\sim} \sim$ 은ํำ

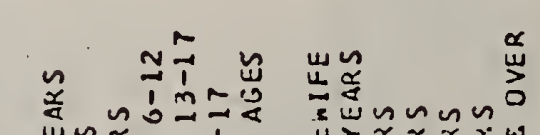
wnศ̆

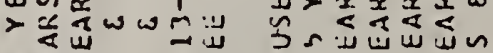

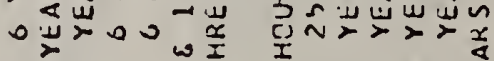
$x^{2} \sim x x^{\infty} \pm$

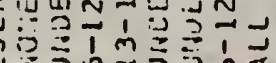

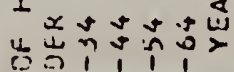
แง

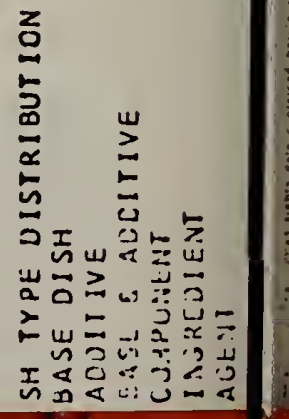


|

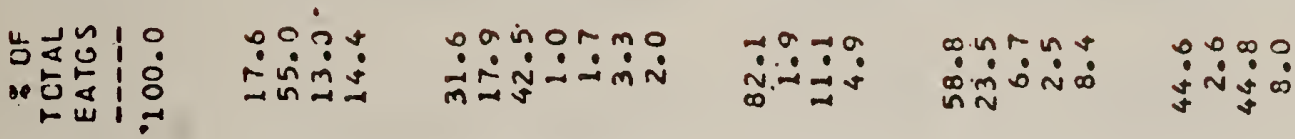

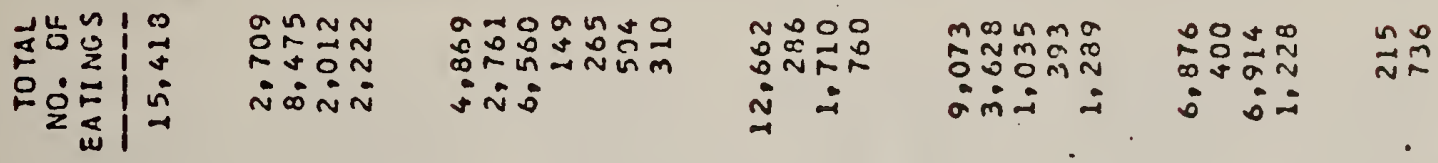

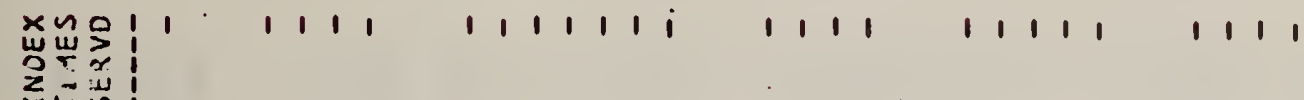

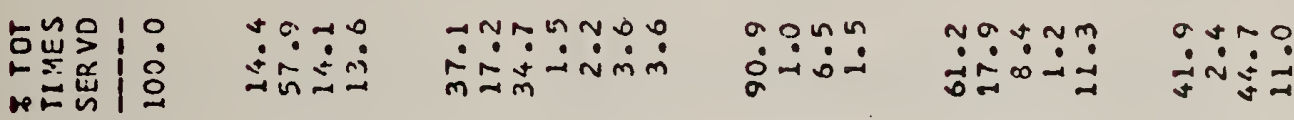

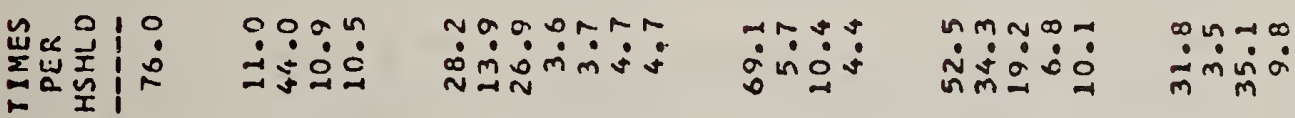

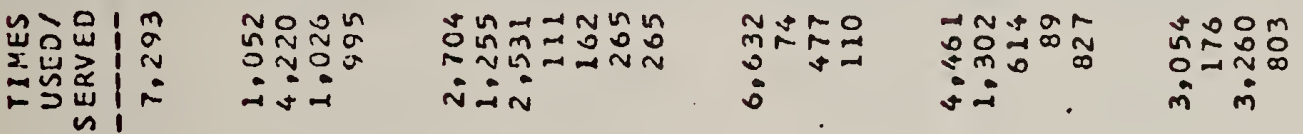

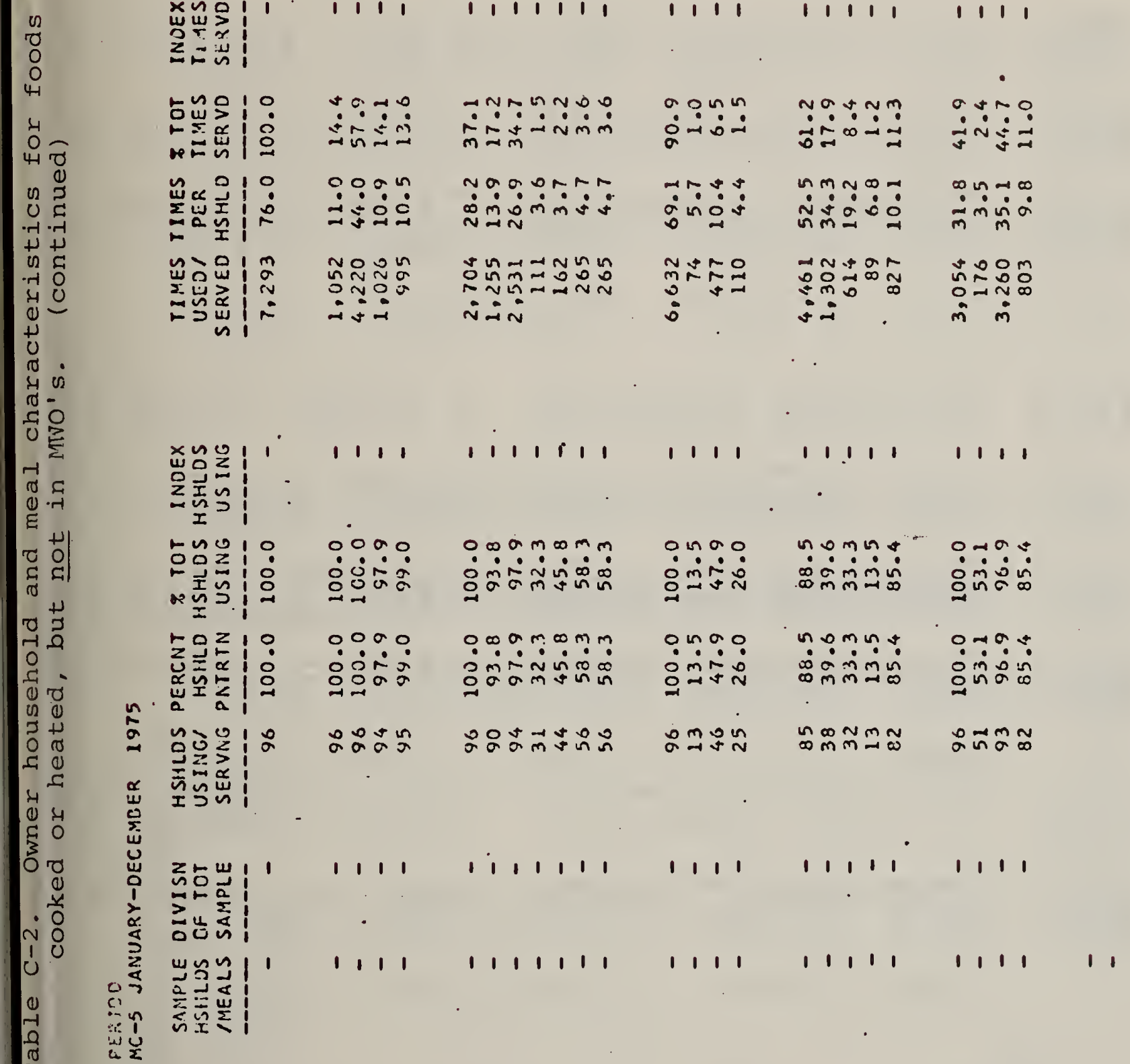

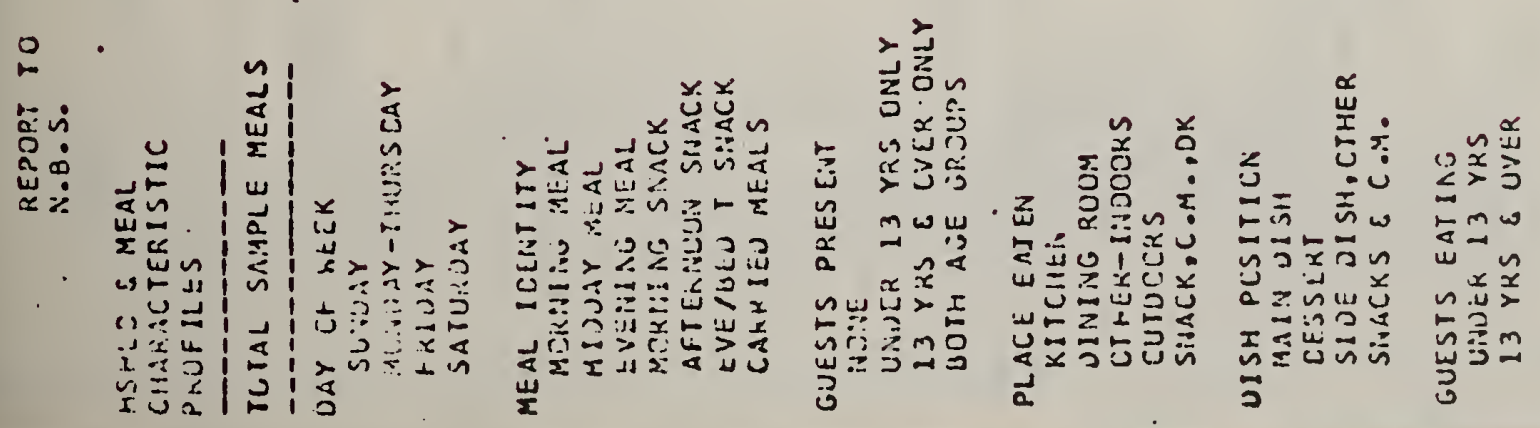


(2)

$\cdot+1 \cdot+$

u

- 40

(1)

$+$

$\infty$

म in

ช્ટ

तु

(1) $\cdot r$

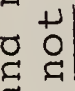

त

ठ

O

(1)

ט 0

כ)

C 0

वृ

w

ㅇํㅇ

i 0

a

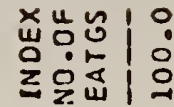

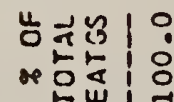

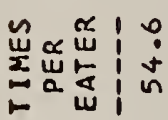

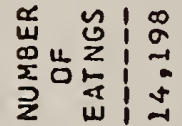

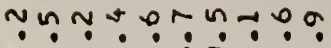

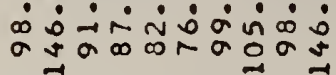

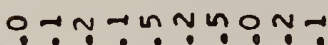

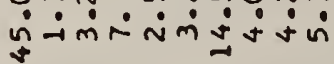

0 O

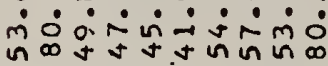

ง

$\begin{array}{lll}0 & 0 \\ 0 & 0 & 0\end{array}$

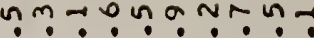
- $\dot{0} \dot{0} \dot{0} \dot{0} \dot{0} \dot{0}$

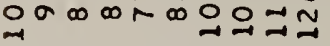

OONT $\infty \infty N m m$

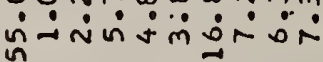

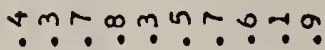

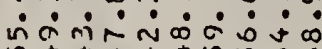

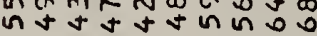

$\pm \infty 0 m 乏 \pm \infty \approx \infty m$ a $\rightarrow$ m
N I Nmon - $\quad \dot{0} \dot{\sim} \dot{\sim} \dot{\sim} \dot{m}$ a वㅇำ

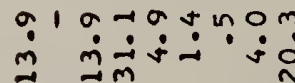

m I mamormin - $\dot{i} \dot{\infty} \dot{\sim} \dot{\sim}$ Nin

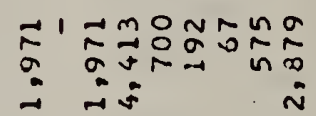

$\infty m a r 0 \infty 1 m u$ ن் $\dot{0} \dot{0} \dot{0}$ वं $m \sim \dot{\sim} \dot{\sim} \dot{\sim}$ i

$\sim \infty+\sigma ? \div$ ? $\dot{n} \dot{i} \dot{0} \dot{0} \dot{N} \dot{a}$

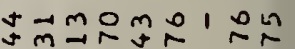

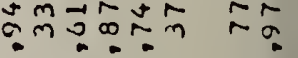
$\rightarrow$ in

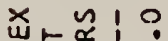
足出出

0000000000 웅 $0 \dot{0} \dot{0} 00 \dot{0} 0 \dot{0}$

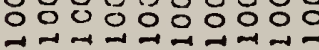

थ 10 은 mon $\rightarrow \cdots n \infty \infty n$ m

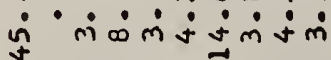
$\alpha z 10$

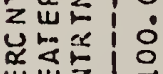
点出云 |

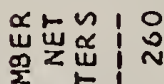
0000000000

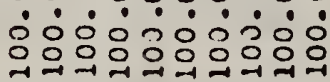

an

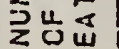

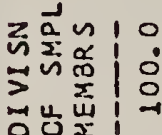

$\underset{\sim}{\infty} \dot{m} \dot{m} \dot{m} \dot{m} \dot{j} \dot{\sim} \dot{\sim} \dot{m}$

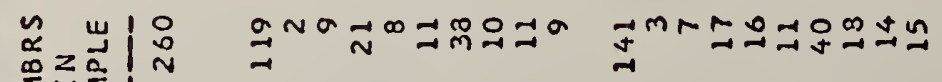

0.00 .0 .000

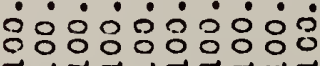

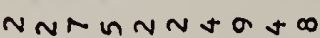

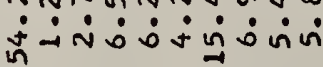

\section{0}

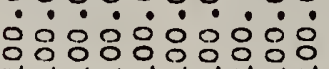

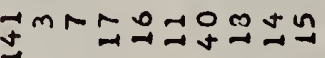

NNRMNNTa+m

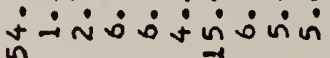

$217 t 0 u t h 0$ nimja 2. 010000000 -

이를

. $100 \% 000 \%$

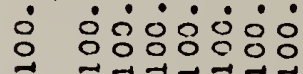

a tam tano

$\rightarrow \lim ^{\circ} \dot{a}$

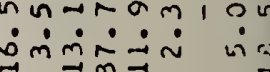
000000100

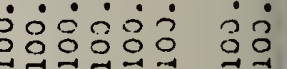
ตु॰

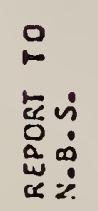
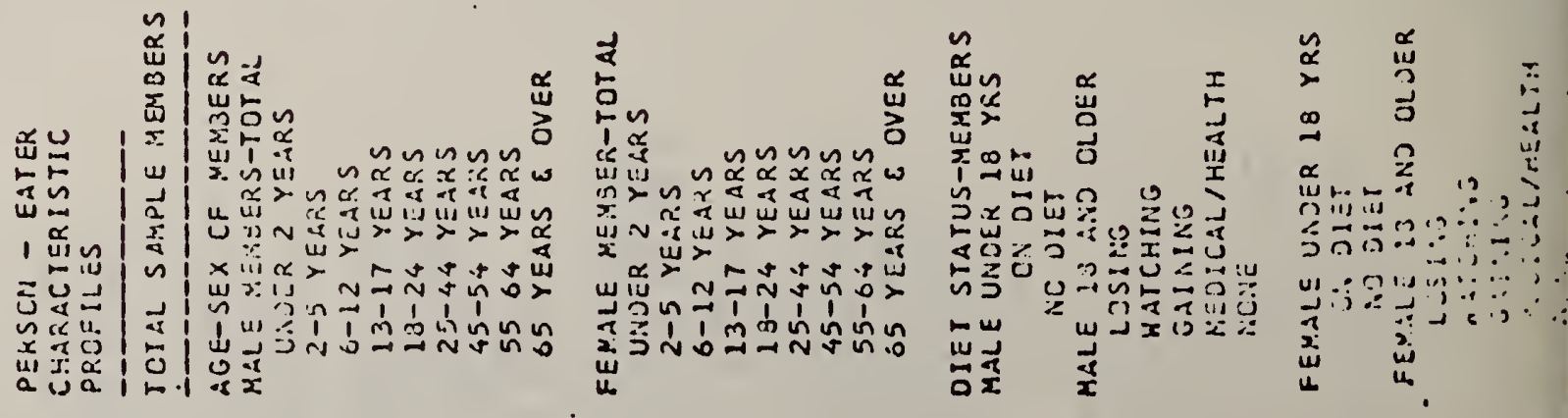


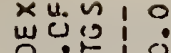
20 व

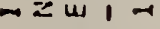

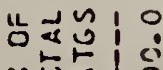

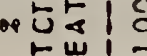

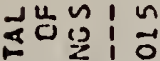
은하

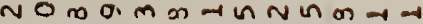

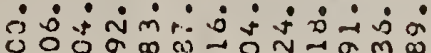

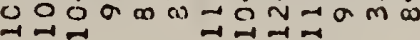

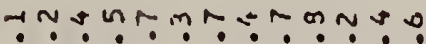

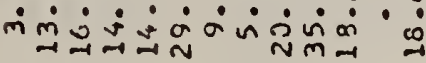

$m \forall r a \sigma \infty 00 \vec{m} m \vec{r}$

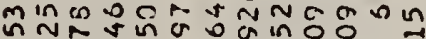
NंÑNi mím

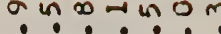

í $\dot{0} \dot{m} \dot{0} \dot{0}$

. $200 \ldots$

namann

ஸ்

$\sim 000 m 0$ का in - $\checkmark v+a \operatorname{man}$

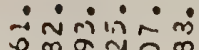
$\therefore \rightarrow$

$\because \because \dot{\sim} \dot{\sim} \dot{m}:$ $\rightarrow \sim N m$

$\operatorname{cana+2~}$

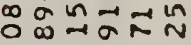
inisin
?. a) $\rightarrow$

$.0 \infty$

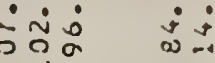

a $0 \div \div \div$

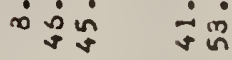

$\sim N 0$ \% $\therefore$ त

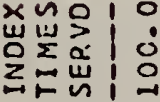

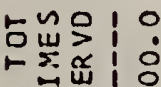

尼出|:

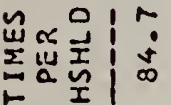

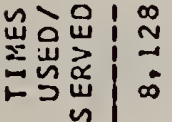

$0 \rightarrow \backsim N a \omega m$ u $\dot{0} \dot{0} \dot{0} \dot{0} \dot{0} \dot{0} \dot{\sim} \dot{\sim} \dot{\sigma} \dot{\sigma}$ $\sigma 00$ क

$\because \cup \sim \backsim \sim m$ in $0 m+m m \sim$

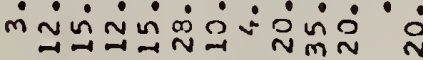

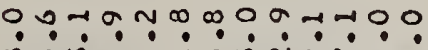
ம் งก๊์ NOVONVD in 0 W aiman ma? 0 ma

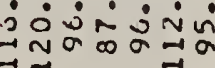

$0 m a r 0 \%$ $\dot{m} \dot{m} \dot{m} \dot{m} \dot{0} \dot{\sim}$

$\neg m \infty m \backsim 0 \infty$

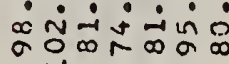

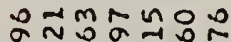
NOOND $\infty$

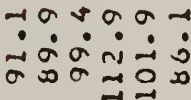

$\ln \ln a \infty N$ $\dot{\sim} \dot{m} \dot{i} \dot{\sim}$ $\rightarrow \sim N$

$\rightarrow n N O N a$

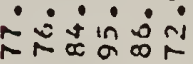

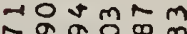
几 00 in in $\begin{array}{ll}m \sim m & +0 \\ \forall \dot{m} & \dot{m}\end{array}$

$\because ? \div$ a

$\because m \infty \quad \div ?$ नूล

a)

$\sim \sim n$

mi mis
兹合识| 呈素出 เ幺ษ 10

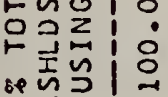
I

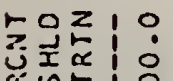

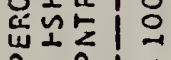
品品

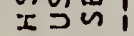

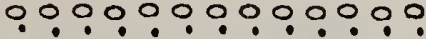

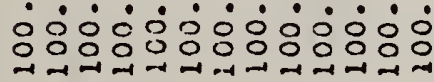
TUMURMmNRNDO

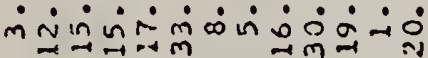

0000000000000

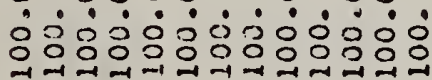

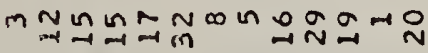

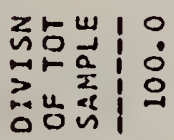
แูป |

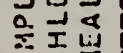

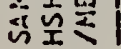

- n? 0 ? m N N N 0 \%

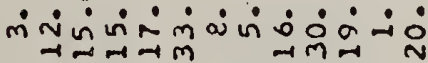

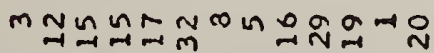

0.000 .0

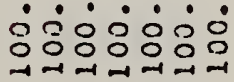

$\operatorname{mannta0}$ $\dot{m} \dot{m} \dot{m} \dot{0} \dot{0}$

0000000

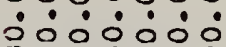

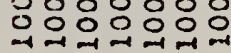

mamsorg

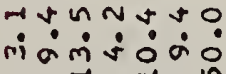

$\sin \pi \mathrm{mm}$ ómi்

.9.0\%०

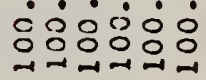

0.0 웅

00

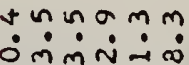
$m \propto 0$ $\omega \dot{j}$

00 에메 $\rightarrow$

000000 0ं $\dot{0} \dot{0} 0 \dot{0}$ 000 ㅇㅇㅇㅇ

00

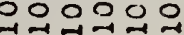

음ำํำ $\infty m i n g$

$m a m+0 a \alpha$

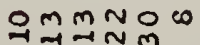

$m \infty a$ $\dot{0} \dot{0}$

00 ai $\infty \underset{j}{j} \tilde{m}$

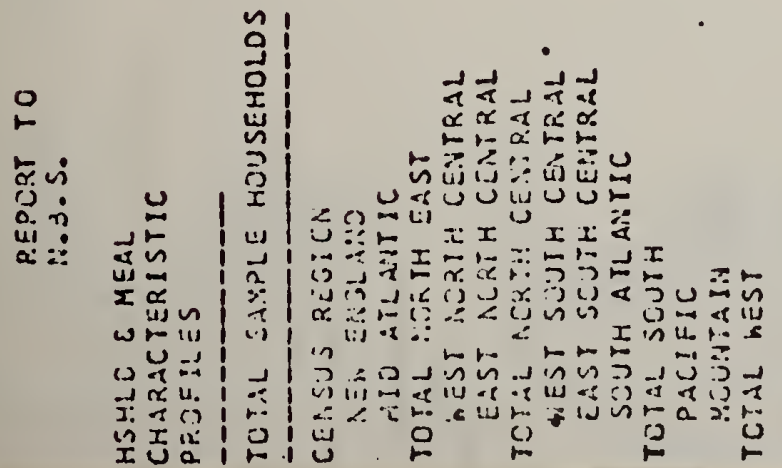

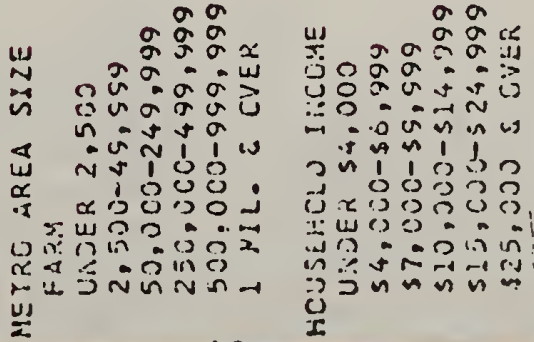

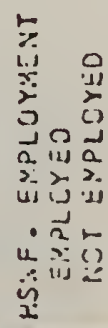

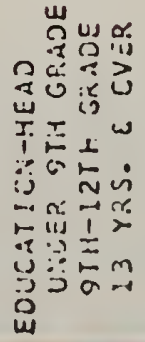




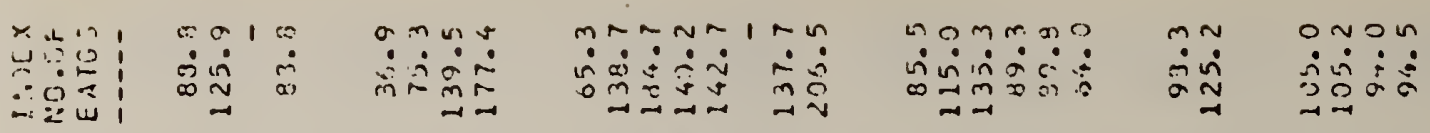

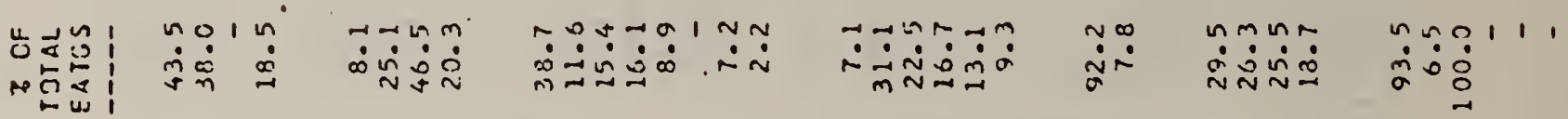

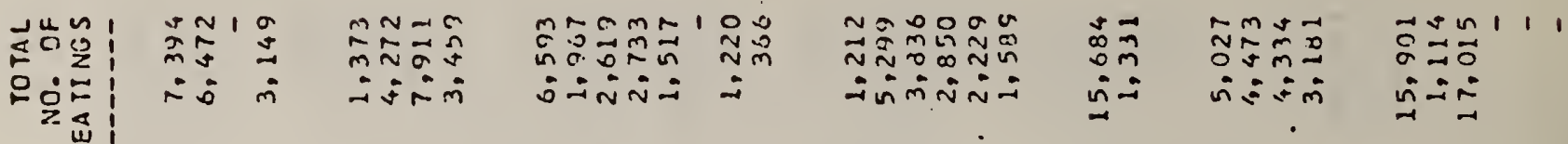



宅

$\frac{1}{\pi}$

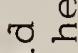

\&

on

2

בิ

40

(1)

है

30

1 C

帘

m

is

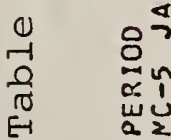

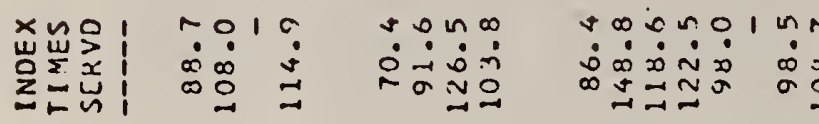

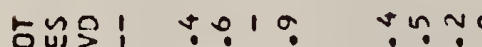

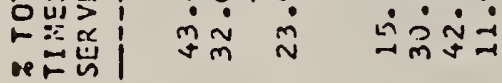

出我!

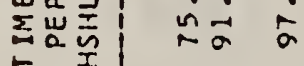

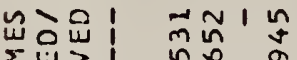

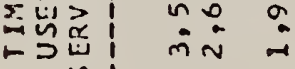

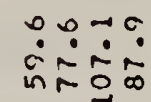

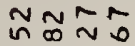

กิบ

$\because \dot{m}$

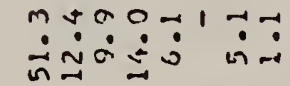

กㄴ?

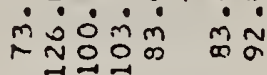

बำ

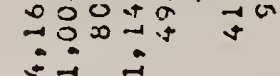

$\sim \sim \infty \infty N 0$

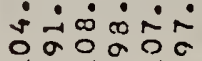

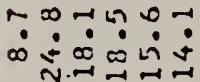

แกーน⿻ $\dot{\infty} \dot{\sim} \dot{m} \dot{0}$

$\infty a+0 \pi 0$

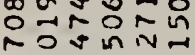

जन

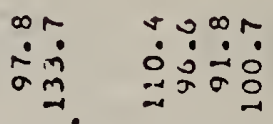

$\because \div \div \%$ á

金

åd

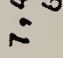

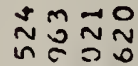

in 200

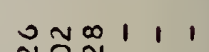
บ?

$\infty \sim 0011$ a $\circ 0$

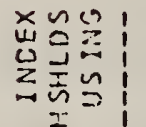

$\begin{array}{lll}0 & 0 & 1 \\ 0 & 0 & 0\end{array}$

?유:

ப요

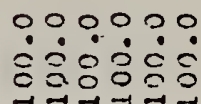

00

$0.0 \%$

c0ㅇㅇㅇ

เn以 1 ON

든ㄹ

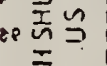

ச்

0010

용 웅

$\therefore 0: 0$

导主并

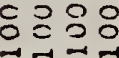

엠ㅇ

$m m n$

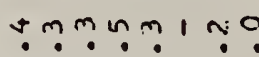

$m-n \infty \infty$

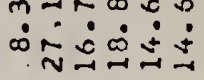

$\ddot{\sigma}$

000000

00000100

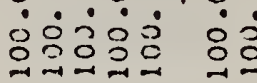

ㅇㅇㅇㅇㅇ

$\infty \circ 0 \infty v t$

กั

$\vec{N} N$ N

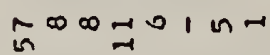

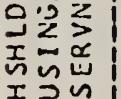

そち山

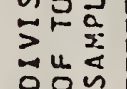

ON10 ammin

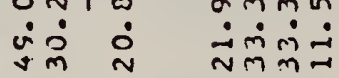

งmm nm 1 ก?

in $\infty \dot{0} \dot{0}$ i

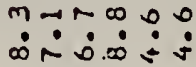

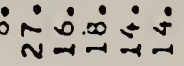

$a$

$\rightarrow 0 \rightarrow \infty$

ஸ் เ⿱丶万仒์

on 0111

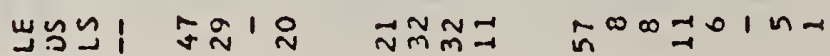
更通

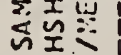

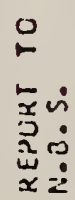

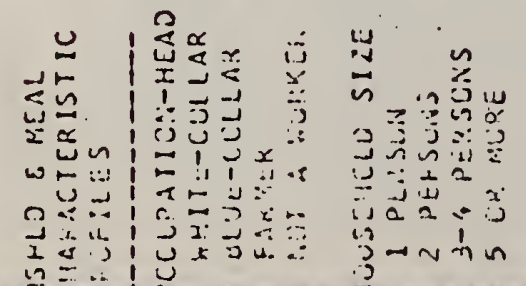

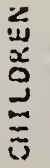

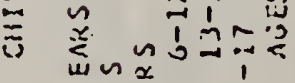

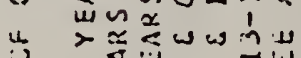

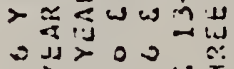

لj

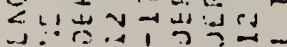

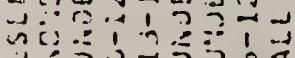

$\infty \stackrel{\sim}{0} \stackrel{\infty}{*} \pm$ 으늄ำ

00

\section{0

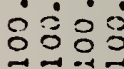

$00 \quad \pi n=9$
○े

111111 $\circ \underset{\infty}{\infty} \approx 111$ 


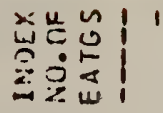

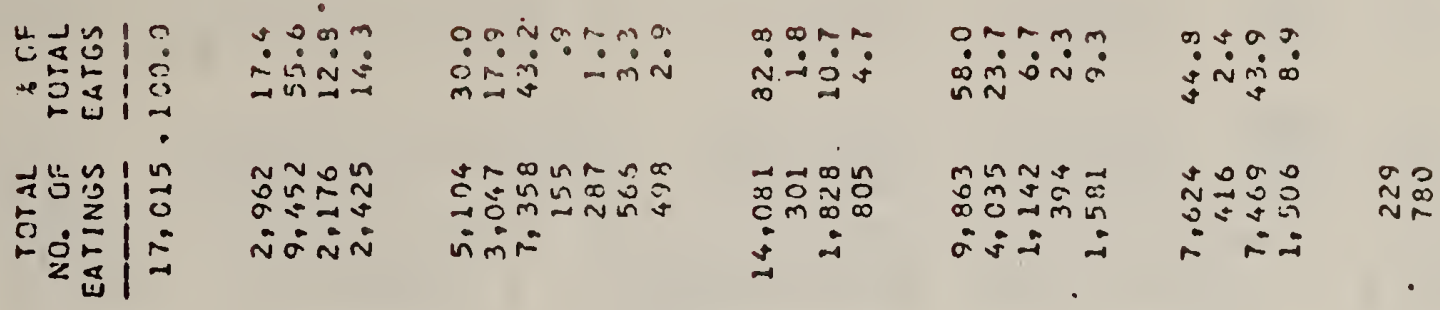

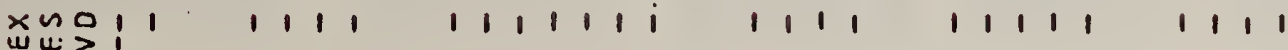

道焉

0

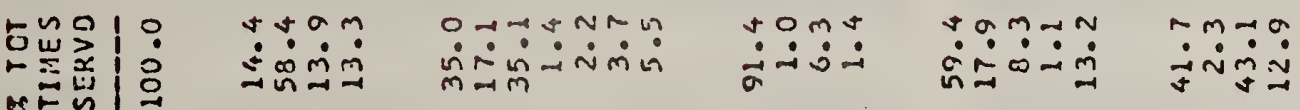

लिए जा

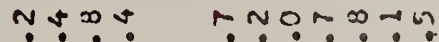

뜬

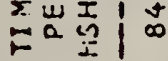

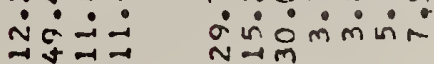

$+\infty \infty \infty$

$\infty \pm+0 ? m u n$

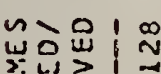

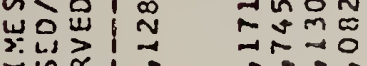

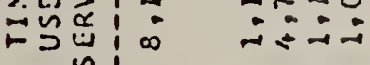

ming

minas

$\operatorname{rin} \dot{0}$

in $\ddot{m} \dot{0} \dot{N}$

$\dot{m} \dot{m} \dot{m} \sim$

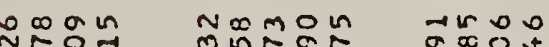

: 政 या

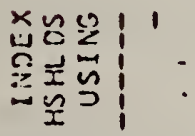

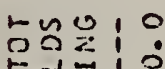

西焉:

0000

0 oomann

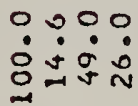

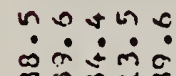

cNa?

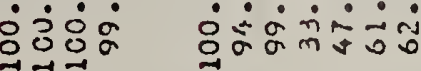

कालिक्ष

8 ถูง

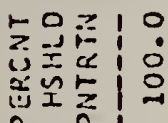

0000 poomman

$\because: 0:$

$\because 0 \pm n 0$

ONOO

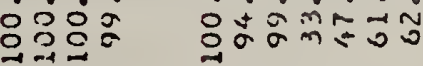

主寺京

$\propto \dot{\sigma} \dot{\dot{m}} \dot{\sigma}$

这官官

ज小 !

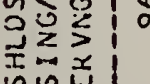

ํํㅇำ ลำ

ถังษณ

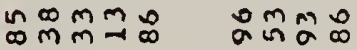

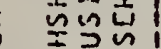

감

$\dot{m}$

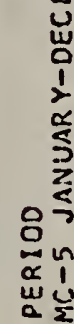

z出!

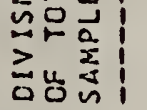

แกะ 111

구의

足出

ถูก๊

1111111

1111

1 1111

1111
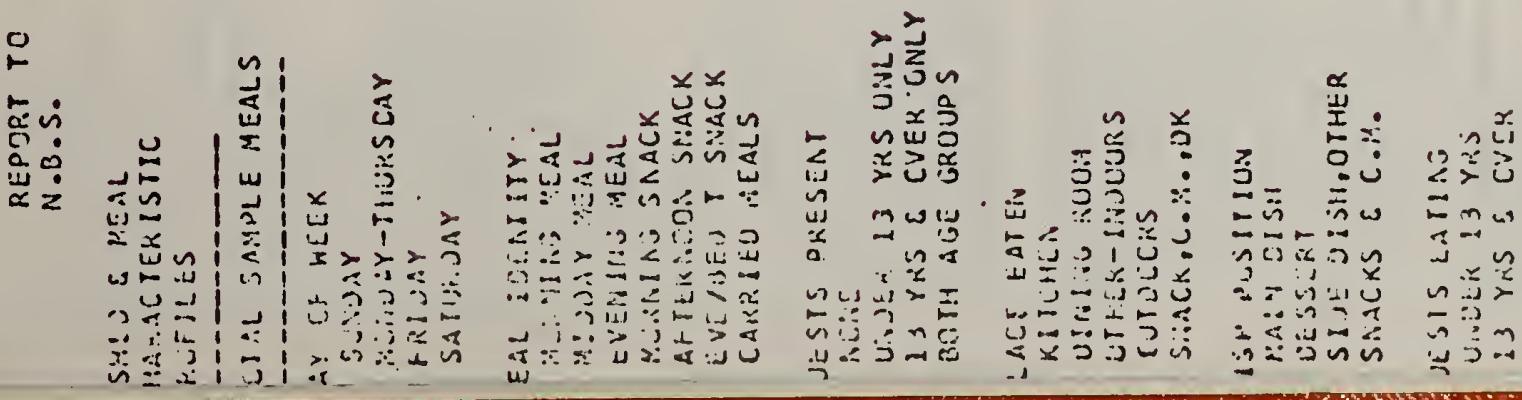


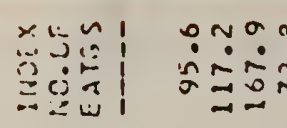

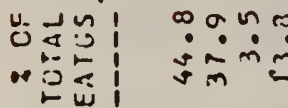

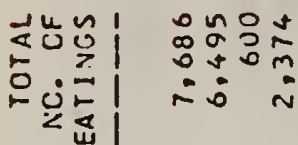

no: 0

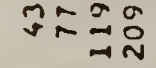

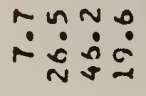

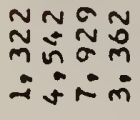

$\infty \rightarrow+\infty \infty|m|$ ํํ용 $\dot{0} \dot{m} \mathfrak{m}$

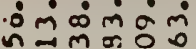

noOnn U U

ปูน

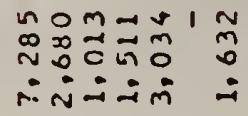

$\sim \sim 0$
Non

manm I

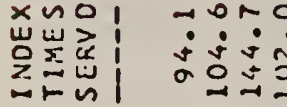

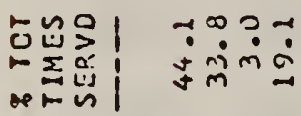

$0: 0$

$\checkmark \dot{\sim} \dot{0} \sim$

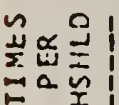

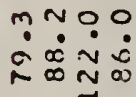

$n>0$

w.

을

in

in -

บ พ บ.

뭉

으웡ㅇㅇ

in்

Noñ்

$\infty \div \infty \div 01 \div$

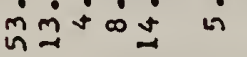

$\infty_{0}: ? \div, \infty 1$

ヘペロすの

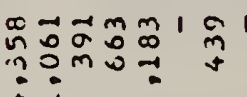

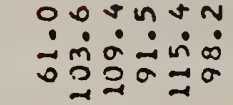

ษ บับำ

กง

ถัก๊ำก

웅웅요는

i

N-1

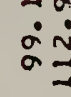

an

:̊n

U

\%)

है

ช

藏

$\nabla \stackrel{1}{=}$

는

(1)

บ2

돈

4

$\begin{array}{ll}0 & \\ 1 & 0 \\ 0 & 0 \\ 1 & 0 \\ 0 & 4 \\ 0 & \\ 2 & \end{array}$

$\begin{array}{lll}ن & 4 \\ 1 & 4\end{array}$

告

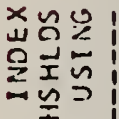

0.00

웡음

เू⿻ $a m-\infty$

o证要

$\dot{q} \dot{\sim} \dot{\sim} \dot{\omega}$

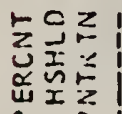

0000

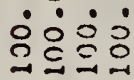

\section{a.}

$\underset{\sim}{n} \sim \mathbb{m}$

能客!

过

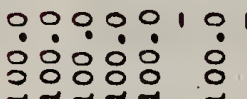

n+us

$\dot{\sim} \dot{m} \dot{m} \dot{0}$

monmUIN

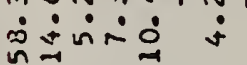

0000

ஓं $\dot{0} \dot{0} 0 \dot{0}$

00000101

용요.

$\simeq m \sim_{m}^{\sigma}$

induro $1+1$

$\because \quad n \because \ln$

$\begin{array}{llllll}1 & 1 & 1 & 1 & 1 & 1\end{array}$

๙

$\tan \pi$

$\dot{\sim} \dot{\sim} \quad \dot{\sim} \dot{N}=\dot{m}$

$\dot{\sim} \dot{0} \dot{0}$

nO NNAB NOnI 1

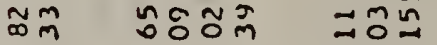

ñ

\section{는면}

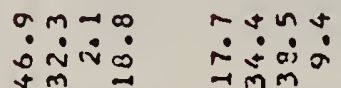

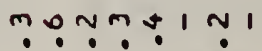

mบขก แ

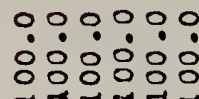

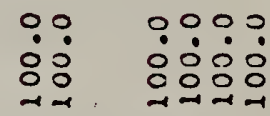

药

nin $\quad 0: \frac{7}{n}$

inai

$\rightarrow$

证落

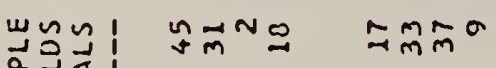

minnio

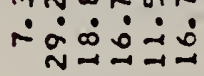

$\stackrel{m}{m} \dot{m}$

$\stackrel{n \sim ⿻}{\sim \sim ⿻}$

ono 111

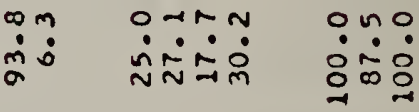

무일

iีะnro⿱

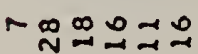

ำ ำก

$\begin{array}{ll}\circ 0 & 0000 \\ \circ 0 & 0000 \\ \circ & 0\end{array}$

$1,111.1$ ㅇㅇㅇㅇㅇㅇㅇㅁㅇ

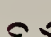

$\stackrel{\sim}{\sim} \beth$

ㅇำ

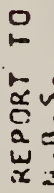

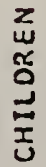

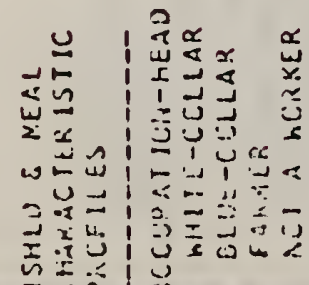

$\stackrel{u}{\Perp} \cong \quad \vec{J}$

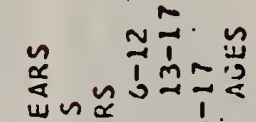
u ग v 过出

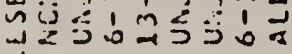

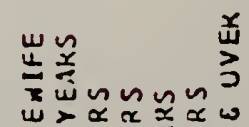

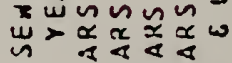

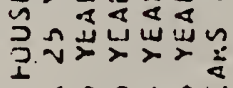

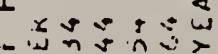

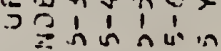

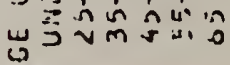

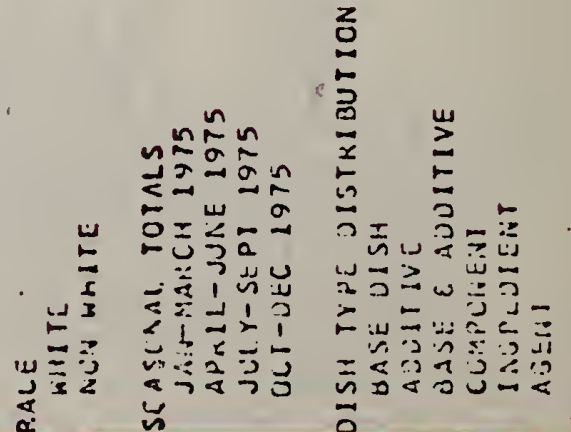


一正山

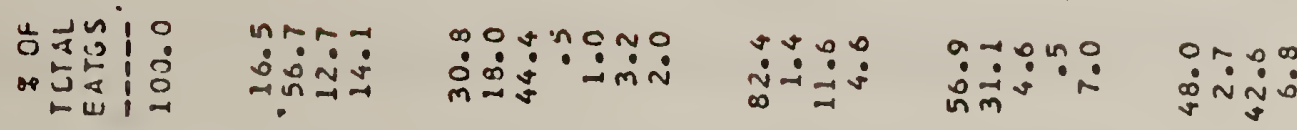

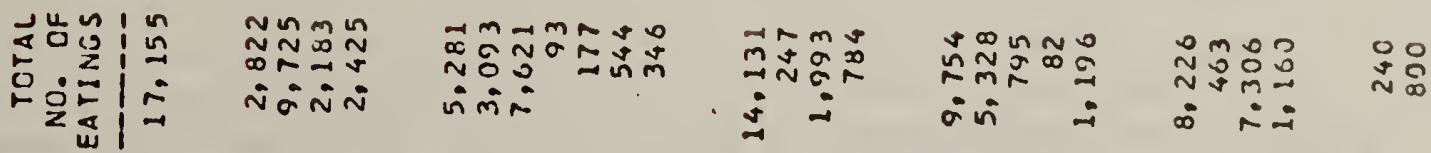

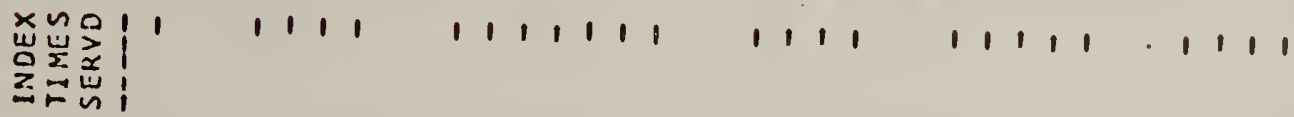

थ

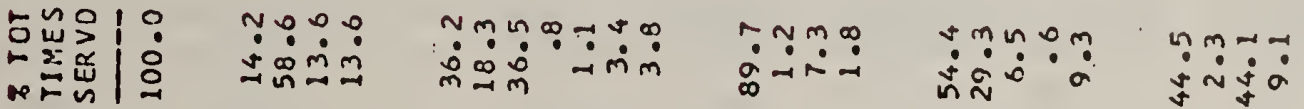

㟧㟧至|

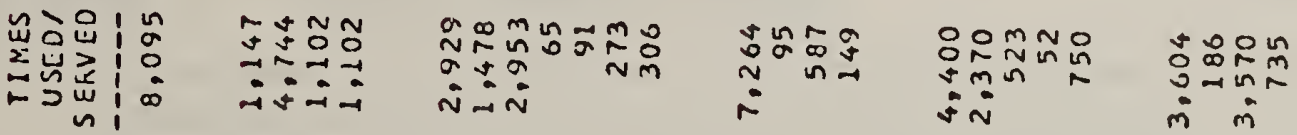

r

$\stackrel{\pi}{\mathbb{1}}$

(ֶ)

$\nabla \stackrel{0}{0}$

苗

ช

어 4

(1)

o

.

됴응

40

(1)

\begin{tabular}{ll}
0 \\
1 \\
0 \\
0 & 0 \\
1 & 0 \\
\hline
\end{tabular}

द $4-1$

Z

2.

نं

is 4

(1)

道

E-1

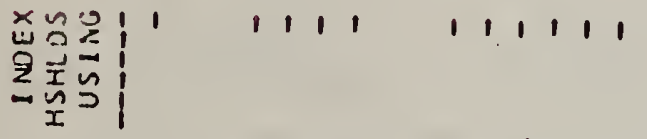

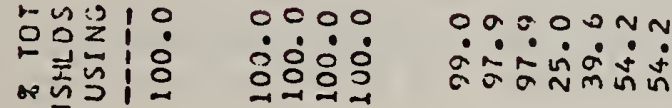

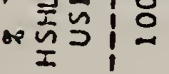

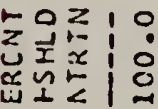

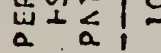

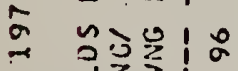

空空蛋

迹会出

羟:

出: z는ㅁ 1

든혀

岩㟧!

m㖑!

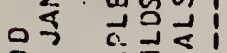

ㅇ

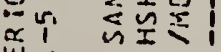

w

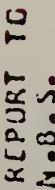

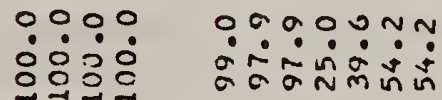

응으

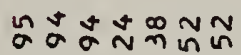

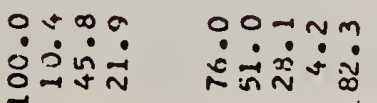

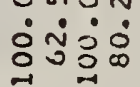

$0+\infty 0$

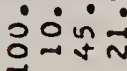

$\because 0 \div \div$ ?

OnON 둥ㅇㅇ

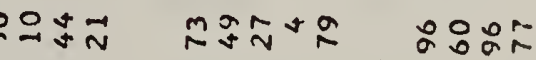

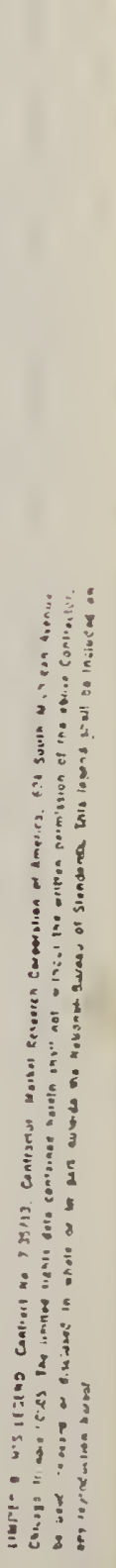


訤峜号|

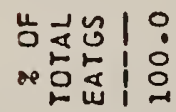

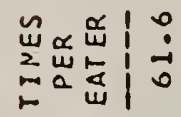

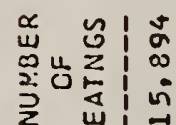

पे

(4)

0

H.

艺

r

. है

.

$+$

ช

-1

단

(1) 0

20

임

1
40

(1)

我

30

द 4

,

Z

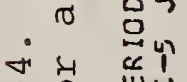

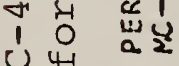

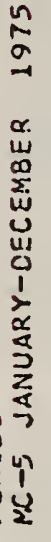

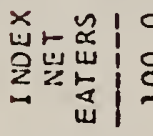

$\infty \infty \infty \infty \infty \infty \infty \infty$

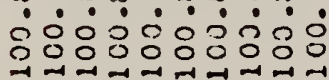

म上 थ 10

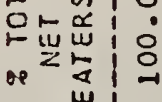

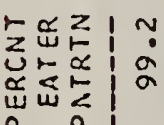

$\infty, \infty, \infty$

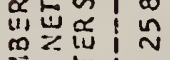
实出告

$z \vec{a} \backsim 10$

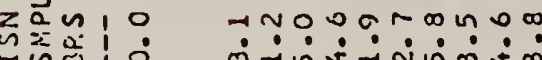

政|

牙岩崖|

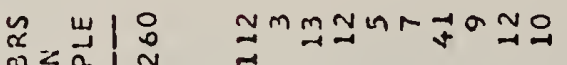

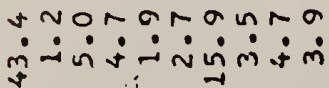

0000000090

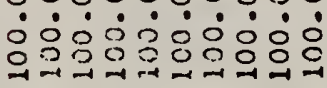

$\cong m m \cong n \pi \exists \sigma \cong 0$

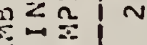

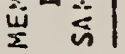

กำง

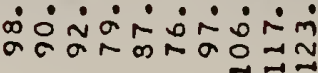

$0 \because \sigma ? \div \because \sigma \div m$

$\dot{\sim} \dot{\sim} \dot{m} \dot{\sim} \dot{\sim} \dot{\sim} \dot{0} \dot{\sim}$

o mun motmom

- $\dot{\sim} \dot{0} \dot{\infty} \dot{m} \dot{\sim} \dot{\sim} \dot{\sim} \dot{\sim}$

o co ins in ton

ป艹

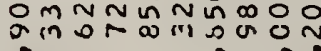

$\infty$

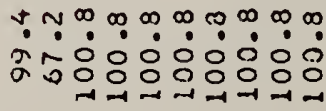

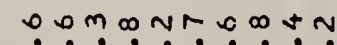

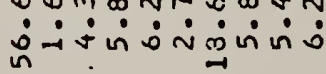

Oro0000000

م

ปัง

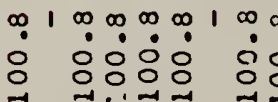

$\because \because \sim \infty \infty \infty \infty \infty \infty$

N 1 in $\infty \pm 1+\infty$

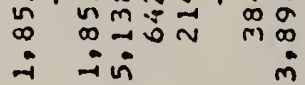

NONNDO요

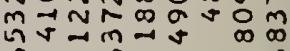

$\ddot{N} \dot{i} \dot{N} \quad \dot{N}$

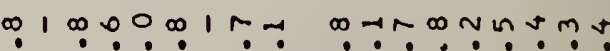
$\stackrel{\dot{\sim}}{\sim} \dot{\sim} \dot{\sim} \dot{\sim} \dot{N} \check{\sim} \dot{\sim} \dot{m} \dot{m} \dot{m} \dot{\sim}$

0.0000100 m.t000000 응ㅇㅇㅇㅇㅇ

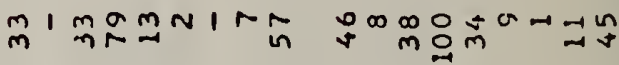

am

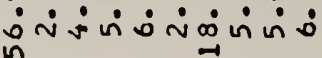

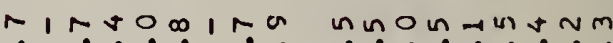
$\stackrel{\sim}{\sim} \dot{\sim} \dot{m} \dot{\sim} \dot{\sim} \quad \dot{m} \dot{\sim} \dot{m} \dot{m} \dot{\sim} \dot{v}$

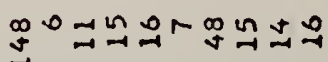

$m \perp m a m n 1-\hat{n}$

क

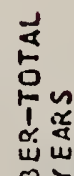

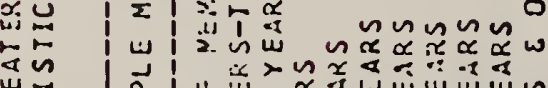

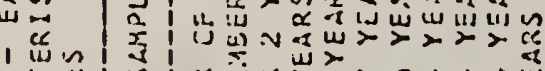

出

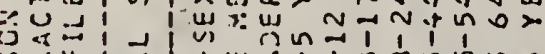

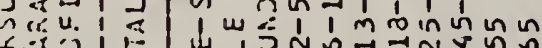
in

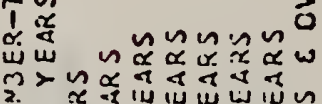

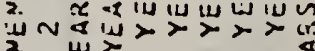

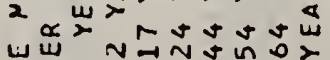

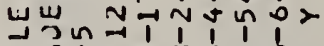

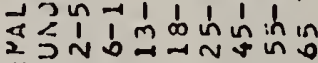

点

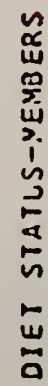

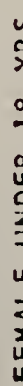

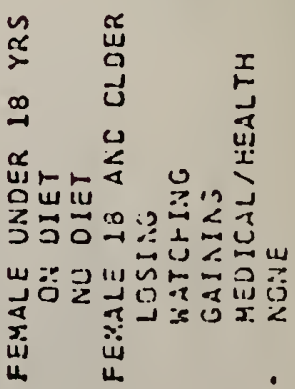

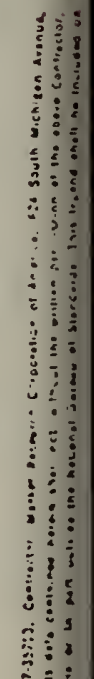

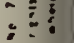

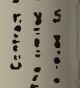


The letter in this appendix from MRCA to NBS is the written release for publication of the particular data in this report for which MRCA reserved limited rights in data. The page numbers in the letter do not correspond exactly to those in the published report because of pagination changes between the version that MRCA reviewed and the present report.

The contractor did not declare Limited Rights in Data on this contract with respect to MWO candidate foods (Report A), but did reserve these rights with respect to the "all foods" data (Report B). The contract between MRCA and NBS includes the following section.

\section{Article $\underline{x}$ - Limited Rights in Data}

1. Notwithstanding Clause 3 of the additional general provisions $\mathrm{PD}-\mathrm{GP}-7 \mathrm{~A}(1-73)$ of this contract, because of the confidential nature of certain data to be made available by the contractor hereunder, the Government (NBS) will take all reasonable precautions not to disclose or otherwise make available to any other firm, person or corporation, any report, analysis or other data, made available hereunder, or extracts therefrom, without the prior written consent of a duly authorized officer of the contractor's firm.

The Contractor agrees that such consent will not be withheld where disclosure is of data at a reasonably high level of aggregation, such as Total Baked Foods, Total Hot Dishes, Total Frozen Prepared Dishes, and the like.

2. Provided that only the data to which limited rights are to be asserted pursuant to paragraph 1 above are marked with the legend below:

\section{LIMITED RIGHTS LEGEND}

Contract No. 7-35713

Contractor: Market Research Corporation of America 624 South Michigan Avenue Chicago, Illinois 60605 
The limited rights data contained herein shall not, without the written permission of the above Contractor, be used, released or disclosed in whole or in part outside the National Bureau of Standards.

This legend shall be included on any reproduction hereof.

NBS has received written permission to release for public distribution all data from Report B contained in this NBS report. 


\section{Market Research Corporation of America}

August 24, 1978

Dr. John V. Fechter

National Bureau of Standards

Department of Commerce

Human Factors Section 441.02

Room A353, B1dg. 220

Washington, D.C. 20234

Dear John:

Thank you for sending to me a draft report dated August 8, 1978 titled

"Houehold Microwave Oven Use", written by Alan D. Davies and John V. Fechter, and for pointing out those sections of this draft which use data provided to you under Contract 非-35713, Modification No. 1, contained in Report B, which is subject to the non-disclosure provisions of this Contract.

Pursuant to your request, permission is hereby granted by MRCA to the National Bureau of Standards to disclose to the public only that information, data, and copies of actual tables already incorporated into the draft copy of the report which you had sent to me.

This authorization for disclosure covers specifically the information shown from Report B in Table 1 on page 11; in Table 2 on page 14; in Table 3 on page 16; in Table 4 on page 19; in Table 5 on page 20; in Table 6 on page 21; in Table 7 on page 23; in Table 9 on page 25; in Table 10 on page 27; in the Contingency Table of Appendix B on page 42; the Demographic Profiles Table C-2 covering all foods cooked or heated by MWO owners, but not in the Microwave Ovens, pages 51, 52, 53, and 54; and the Demographic Profiles for all foods cooked or heated by MWO-owning households shown in Table $\mathrm{C}-3$, pages 55, 56, 57, and 58; and the Demographic Profiles of all foods cooked or heated by non-owners of Microwave Ovens, shown in Table C-4, pages 59, 60, 61, and 62 .

Since Tables $\mathrm{C}-2,-3$, and -4 contain the non-disclosure legend of this Contract, and since the information and data shown in the tables sited above are also subject to the same non-disclosure provisions, a copy of this letter of authorization to disclose this material to th public should be incorporated into your report.

Sincerely yours,

MARKET RESEARCH CORPORATION OF AMERICA

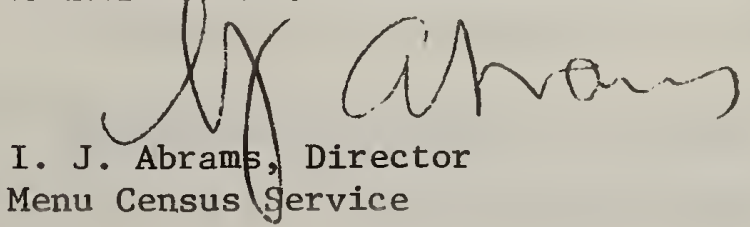

IJA:mak 
US

birlivgriaphic uata

ShIt 5

4. 1111.1. A $(1) 1), 1: 111111.1$

HOUSEHOLD MICROWAVE OVEN USE

N1:!I? 7n-1,7.1?

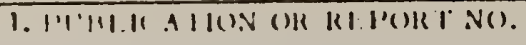

3. Kecipient's Aicessim V.

5. Publicacion lisc

6. Performing Organization Cos

8. Performing Organ. Report N

10. Projece/ Task, hork Linit:

7624422

HATIONAL SUREAU OF STANDARDS

DEPARTMENT OF COMMERCE

11. Contsact/ visant io.

WASHINGTON, D.C. 20234

12. Sponsoring (hy.nization Nume and Complete Ajuress (Street, City, State, ZIP)

National Bureau of Standards and

office of Conservation Covered

Final

Department of Energy

Washington, D.C. 20461

14. Sponsoring $A_{\text {gency Code }}$

15. SUPPLEMENTARY NOTES

16. ABSTRACT (A 200-word or less factual summary of most significant information. If document includes a significant bibliosraphy or literature survey, mention it here.)

The National Bureau of Standards (NBS) has been recommending test methods for measuring the energy efficiency of major household appliances. Part of this work involved comparing the usage of microwave ovens (iwO's) with that of other products for cooking or heating foods.

From a 1975 national survey of 2000 households, Market Research Corporation of America selected 96 households in which an 1 ItO had been used to prepare foods (i-10 owners), and a demographically matched set of 96 "non-owner households. Data on Servings (dishes prepared) and Eatings (persons partaking of a Serving) were provided under contract to NBS. The main results were:

- There was little difference between the owner and non-owner households in terms of total Eatings or Servings or in the proportions of leftovers eaten.

- In owner households, MH's accounted for approximately 10 percent of the Servings and 9 percent of the Eatings.

- Leftovers accounted for 9 percent of the Eatings for both groups, and for 11 percent of owner Servings and 9 percent of non-owner Servings.

- MN's were used by owners for 26 percent of leftover Servings and 23 percent of leftover Eatings.

17. KEY WORDS (six to twelve entries; alphabetical order; capitalize only the first letter of the first key word unless a proper name; separated by semicolons)

Appliance efficiency; consumer survey; cooking and heating; energy; food preparation; leftovers; microwave ovens

\section{AVAILAIILITY}

For Official Distribution. Do Nor Release to NTIS

Order From Sup. of Doc., U.S. Government Printing Office Washington, D.C. 20402, sl) Siock Nu. S.v(10)3-(10)

Order From Nntional Technical Information Service (NTIS) Spinklicld, Virginea 22151

\begin{tabular}{|l|l|}
\hline $\begin{array}{l}\text { 19. SECURITY CLASS } \\
\text { (THIS REPURT) }\end{array}$ & 21. NO. OF PAGLS \\
UNCL ASSIFIED & \\
\hline $\begin{array}{l}\text { 20. SI:CURITY CLASS } \\
\text { (THIS PAGE) } \\
\text { UNCLASSIFIED }\end{array}$ & 22. PriCC \\
\hline
\end{tabular}

